\title{
Linking audiovisual integration to audiovisual speech recognition in noise
}

\author{
1 Anja Gieseler ${ }^{1,5, \#^{*}}$, Stephanie Rosemann2,5, Maike A. S. Tahden ${ }^{1,5}$, Kirsten C. Wagener ${ }^{3,4,5}$, \\ 2 Christiane M. Thiel2,5, Hans Colonius ${ }^{1,5}$ \\ ${ }^{1}$ Cognitive Psychology, Department of Psychology, School of Medicine and Health Sciences, Carl \\ von Ossietzky University Oldenburg, Oldenburg, Germany \\ ${ }^{2}$ Biological Psychology, Department of Psychology, School of Medicine and Health Sciences, Carl \\ von Ossietzky University Oldenburg, Oldenburg, Germany \\ ${ }^{3}$ Hörzentrum Oldenburg GmbH, Oldenburg, Germany \\ ${ }^{4}$ HörTech gGmbH, Oldenburg, Germany \\ ${ }^{5}$ Cluster of Excellence 'Hearing4all', Carl von Ossietzky University Oldenburg, Germany \\ \# Current Address: Rehabilitation Science, Department of Health Services Research, School of \\ Medicine and Health Sciences, Carl von Ossietzky University Oldenburg, Oldenburg, Germany
}

*Corresponding author

Anja Gieseler

Email: anja.gieseler@uni-oldenburg.de

\section{Abstract}

Especially in challenging listening conditions, listeners can benefit from the audiovisual nature of speech by using visual information. Yet there exists great inter-individual variability, not only in understanding speech in noise, but also in the benefit obtained from additional visual cues. First empirical evidence suggests that the ability to integrate auditory and visual input, i.e. audiovisual integration, is altered in hearing impairment and is, at the same time, relevant for audiovisual speech intelligibility. The distinct role of mild hearing loss on audiovisual integration and the significance of these changes for speech intelligibility, however, need further scrutiny. Thus, here we investigated differences in audiovisual integration capacities between elderly, normal-hearing and hearing-impaired individuals using two tests of audiovisual integration (sound-induced flash illusion, McGurk task). To explore whether potential differences in audiovisual integration are meaningful for natural speech intelligibility, we then linked audiovisual integration capacities to speech-in-noise recognition using an audiovisual speechreception threshold test, expecting this to reflect a more realistic listening scenario. Our results indicate that audiovisual integration abilities are already altered in mild hearing impairment, while the magnitude and direction of the effect depend on the specific test used. At the same time, audiovisual integration capacities seem relevant for predicting audiovisual speech intelligibility in noise, especially in those individuals with a hearing loss. We conclude that audiovisual integration abilities should therefore be considered for future predictions of speech recognition outcomes, which - in turn - should be assessed audiovisually, to account for the multisensory nature of speech and communication.

Keywords: flash illusion, McGurk, hearing loss, audiovisual SRT test, multisensory integration 
Speech intelligibility can be a challenging task with the speed of a conversation reaching up to 200 uttered words per minute [1]. Furthermore, the clarity of the acoustic signal might be compromised - be it due to the characteristics of the speaker (e.g., accent, mumbling), due to the characteristics of the listening situation (e.g., reverberation, background noise), or due to the listener's impaired hearing abilities. Especially older adults with a hearing loss experience increased difficulties in understanding speech in such challenging listening situations. Therefore, listeners not only rely on the acoustic information, but make use of additional information, such as the linguistic context or visual cues from the speaker's face. Decades of research have demonstrated that when additional, congruent visual cues are provided, speech recognition is substantially improved for both normal-hearing and hearing-impaired individuals [e.g., 2-10]. This is reflected in an increase of correctly identified audiovisual compared to auditory-only speech [9], improved signal-to-noise ratios (SNRs) [2, 4, 6], accelerated neural speech processing [11, 12], or reduced listening effort for the listener [13]. Thus, integrating auditory and visual information can improve speech intelligibility, especially in noisy conditions in which visual information can provide an increase in the signal-to-noise ratio to as much as $15 \mathrm{~dB}[2,4]$. This benefit of audiovisual- over auditory-only speech has been termed audiovisual gain or visual enhancement.

While it is well-known that elderly listeners differ greatly in understanding auditoryonly speech in noise ( $\mathrm{SiN}$ ), apparently the same is true for audiovisual speech intelligibility and for the extent of using visual speech information. Accordingly, there exists a large variability among individuals in their benefit from additional visual cues, i.e. their audiovisual gain, even when both auditory-only and visual abilities are taken into account $[9,14-17$, see also 18]. This might be explained by the finding that listeners differ not only in the extent to which they extract the unisensory signals, but also in the degree to which they combine (or integrate) visual and auditory information [14]. Grant and colleagues described substantial inter-individual differences among hearing-impaired subjects in integrating auditory and visual consonant cues and, importantly, found these to be related to the audiovisual gain, such that those who integrated better also showed a larger audiovisual benefit. 
These findings and the fact that the variability in SiN recognition has not been fully accounted for by measures of age, auditory- or cognitive abilities [e.g., 19, 20], have prompted the conclusion that audiovisual integration (AVI), i.e. the ability to integrate auditory and visual input, is a distinct capacity, independent of auditory- and visual abilities, and likewise important for audiovisual speech processing [cf. 14, 18, 21, 22]. Given the multisensory nature of natural speech and communication [cf. 23], this points to the potential role of audiovisual integration abilities in explaining differences in audiovisual speech intelligibility. At the same time, classical assessments for speech recognition or hearing-aid outcomes apply speech-reception threshold (SRT) tests solely in auditory-only conditions. The undisputable role of visual information for speech-innoise intelligibility challenges the assumption that auditory-only SRT tests reflect an ecologically valid listening scenario [cf. 24]. Here, we therefore aimed at investigating the relation between audiovisual integration abilities and audiovisual speech-in-noise recognition.

Different approaches on both neuronal and behavioral levels have been used to measure audiovisual integration [cf. 22, 25]. A common approach is to use paradigms with incongruent audiovisual input that may elicit perceptual illusions necessarily driven by audiovisual integration. Two prominent examples are the McGurk effect [26] and the sound-induced flash illusion (SIFI) [27, 28]. In the prototypical version of the McGurk task, the sound of the syllable /ba/ is presented with the visual image of a speaker articulating /ga/, commonly leading to the fused percept /da/, which is taken as an indicator for AVI. The flash illusion, on the other hand, pairs one flash with multiple beeps, potentially resulting in the illusory percept of multiple flashes, again indicating AVI. Crucially, there exists an individual time interval in which the inputs from the auditory and visual modality are most likely to be integrated, reflected by change in perception or behavior. This time interval is known as temporal window of integration or temporal binding window $(T B W)$, with the latter commonly referring to the stimulus onset asynchronies (SOAs) across which an effect is observed [e.g., 29-32, for review see 33]. Thus, one way to measure the TBW is to vary the SOAs between the stimuli and explore the corresponding perceptual responses. stimuli in McGurk, low-level stimuli in SIFI), but also in the nature of the task and 
104 crossmodal interaction: In the McGurk task, auditory perception might be altered by 105 incongruent visual input, whereas in SIFI, visual perception might be altered by 106 incongruent auditory input. An earlier study found a negative correlation between the 107 two tests in healthy young adults [34]. At the same time, there is converging evidence for 108 distinct neural processes underlying different aspects of audiovisual integration [cf. 35]. 109 Thus, we used both tests to investigate their relationship and their potentially distinct contribution to speech intelligibility in noise

One line of research on multisensory integration in (healthy) elderly adults suggests that they show enhanced audiovisual integration abilities when compared to younger 113 adults [e.g., 36-41], although this has not been found consistently [e.g., 42-44]. 114 Considering the use of various tasks and analysis methods, however, there is good overall 115 agreement on increasing multisensory integration abilities with aging, yet the reasons for 116 these enhanced abilities seem multifactorial [for reviews see 45, 46]. Among others, it is 117 proposed that enhanced integration abilities compensate for unisensory deficits, i.e. for 118 the age-related declines in sensory modalities such as vision and audition. Additionally, 119 although hearing impairment constitutes one of the most prevalent conditions in the 120 elderly, relatively little is known about audiovisual integration in older adults with 121 hearing loss compared to their normal-hearing peers. While there is evidence that AVI 122 alters not only with aging but is also affected by varying degrees of hearing impairment 123 [e.g., 21, 47-49], the separate contribution of hearing impairment to AVI remains to be 124 further explored.

125 Previous studies have largely focused on cochlear-implant (CI) patients with 126 profound hearing loss [for reviews see 50,51], whereas studies of rather mild forms of 127 hearing loss and of the effects of hearing aids on AVI have been rare and just recently 128 complemented. Altieri and Hudock [21] revealed relationships between measures of AVI 129 and hearing abilities, suggesting that the ability to integrate auditory and visual speech 130 input is linked to auditory abilities. Adding to this, there is evidence for increased AVI and 131 altered audiovisual speech processing in mild-to-moderately hearing-impaired compared 132 to normal-hearing, older adults, as indicated by the susceptibility to the McGurk effect 133 [49]. A previous study of ours using the flash illusion extended this to hearing-aid use, 134 finding more AVI in hearing-aid users with a mild hearing loss compared to their 135 untreated peers, who were matched for age and degree of hearing loss [52]. 
In summary, there is evidence for altered AVI abilities in elderly, hearing-impaired

137 listeners and for the potential role of AVI abilities for understanding speech in noise, given

138 the audiovisual nature of everyday-life communication. Nonetheless, the separate role of

139 (mild) hearing loss on AVI and the significance of these changes for speech intelligibility

140 are yet unclear. Thus, here we investigated differences in AVI capacities between elderly,

141 hearing-impaired and age-matched, normal-hearing individuals using two different 142 paradigms, the McGurk task and SIFI. To see whether the potential differences in AVI are 143 meaningful for natural speech intelligibility, we then linked AVI capacities to SiN 144 recognition using an audiovisual SRT test [53], arguing that this reflects a more realistic 145 listening scenario.

146 This study was motivated by three main topics. We aimed at investigating (i) AVI 147 capacities, (ii) audiovisual SiN recognition and the audiovisual gain, and (iii) the relation 148 between AVI and audiovisual SiN recognition in elderly, hearing-impaired (HI) 149 individuals and age-matched, normal-hearing ( $\mathrm{NH}$ ) controls. To that end, we compared 150 those two groups across two tests of AVI (McGurk, SIFI) with respect to their 151 susceptibility to the respective illusion and the corresponding temporal binding window, 152 as well as in their performance in an audiovisual SRT test. Finally, we linked these 153 measures by means of a stepwise linear regression to evaluate the amount of (additional) 154 predictive value of AVI for SiN intelligibility, beyond the influences of age and hearing 155 abilities.

156 We formulated the following three hypotheses:

157 H1) We predict that groups would differ in their AVI abilities as indicated by the McGurk 158 and SIFI, with more McGurk illusions in the HI compared to the NH group and the opposite 159 for SIFI, that is, more illusory flashes in the NH group than the HI group.

$160 \mathrm{H} 2$ ) We predicted that the HI individuals perform worse in the audiovisual SRT test than 161 NH individuals but possibly display a larger audiovisual gain.

162 H3) Finally, we assume that AVI abilities would account for additional variance in 163 audiovisual SiN recognition particularly in the HI group and for the McGurk task, which is 164 more speech-like than the flash illusion. 


\section{Materials and Methods}

166

167

168

169

170

171

172

173

174

175

176

177

178

179

180

181

182

183

\section{Participants}

Twenty-five normal-hearing individuals $(\mathrm{NH})$ with a mean age of $68.8 \pm 5.3$ years, and twenty-five hearing-impaired individuals (HI) with a mean age of $70.4 \pm 6.2$ years, participated in this study. The age did not differ significantly between the groups $\left[\mathrm{T}_{(48)}=-\right.$ 0.929, $p=0.357]$. The hearing-impaired group displayed a mild, symmetric, and agerelated hearing loss (presbycusis) that was typically present in the high frequencies. According to the WHO definition, they had better-ear pure-tone averages across the frequencies $0.5,1,2$, and $4 \mathrm{kHz}$ (PTA-4) of between 26 and $40 \mathrm{~dB}$ HL [cf. 54, 55] with a mean PTA-4 of $31.3 \pm 7.7 \mathrm{~dB}$ HL, whereas the normal-hearing group had PTA-4 values below $20 \mathrm{~dB}$ HL (mean PTA-4 of $9.2 \pm 3.0 \mathrm{~dB}$ HL), with PTA-4 differences between left and right ear smaller than $15 \mathrm{~dB}$ HL and air-bone gaps no larger than $10 \mathrm{~dB}$ HL. Fig 1 shows the individuals' pure tone audiograms averaged over both ears. None of the subjects reported prior hearing-aid experience.
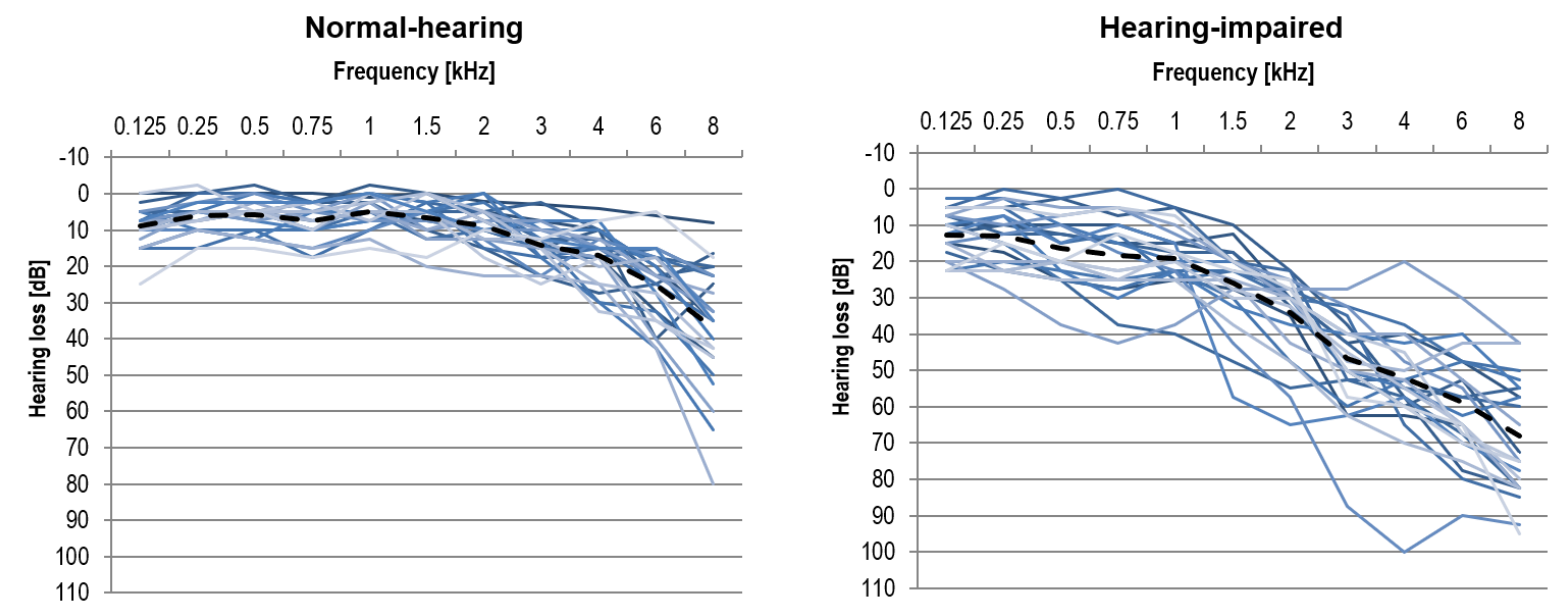

Fig 1: Pure-tone audiograms of the participants. Shown are the individual pure-tone audiograms for normal-hearing (left) and hearing-impaired (right) participants (mean across both ears) as well as group averages (dashed line).

All participants had normal or corrected-to-normal vision and did not show indication of dementia (age-normed DemTect scores of $>$ 8) [56]. The groups did not differ in the DemTect scores [ $\left.\mathrm{T}_{(48)}=-0.877, p=0.385\right]$. Individuals with psychiatric or 
neurological disorders were excluded from the study. The study was approved by the local ethics committee of the University of Oldenburg (Committee for Research Impact Assessment and Ethics) and performed in accordance with the Declaration of Helsinki. All participants gave their written, informed consent prior to the study and received a monetary compensation for participation.

\section{Tests and stimuli}

To assess audiovisual integration (AVI) capacity as well as the corresponding temporal binding window, the test battery comprised two tests, the McGurk-MacDonald illusion (McGurk) and the sound-induced flash illusion (SIFI), with the individuals' susceptibility to the respective illusion taken as an indicator for the strength of AVI and the time frame in which the illusions occurred as an indicator for the respective TBW. To determine speechin-noise intelligibility for auditory-only and audiovisual conditions, we used the Audiovisual Oldenburg Sentence Test (AV-OLSA).

\section{Tests of audiovisual integration}

\section{McGurk}

In the McGurk task, participants saw video clips of a speaker's face and heard spoken syllables, which were either congruent to the lip movement of the speaker or incongruent, which may result in auditory illusions when indicating the syllable heard [26].

As audiovisual stimuli, a subset of syllables by a male speaker, created in the Department of Media Production at the University of Oldenburg, was used [49]. Specifically, we only used the syllables /da/ and /ba/, commonly resulting in the illusory fusion percept /da/ when presented in an incongruent condition (i.e. visual /gav/ paired with auditory /baA/), as previous studies found this combination to be the most robust in eliciting the McGurk illusion in healthy, German-speaking participants $[49,57]$.

The test consisted of seven conditions: Four unimodal conditions involving auditoryonly ( $\mathrm{A}_{\text {only }}$ ) / $\mathrm{baA}_{\mathrm{A}}$ / and /gaA/ as well as visual-only ( $\left.\mathrm{V}_{\text {only }}\right)$ /bav/ and /gav/ with five repetitions each, two audiovisual congruent ( $\mathrm{AV}_{\mathrm{cong}}$ ) conditions (/baA-bav/, /gav-gav/) as well as the incongruent, illusory fusion condition ( $\left.A V_{\text {incong }}\right) / \mathrm{baA}-\mathrm{gav} /$. The auditory and visual stimuli were presented either synchronously (SOA of $0 \mathrm{~ms}$ ) or across SOAs of 70$420 \mathrm{~ms}$ in steps of $50 \mathrm{~ms}$ to quantify the temporal binding window, with the onset of the 
tone following the onset of the video. Each SOA (0, 70, 120, 170, 220, 270, 320, 370, 420

$218 \mathrm{~ms}$ ) was repeated ten times, summing up to 200 interleaved trials in total for the whole test, which lasted approximately 20 minutes.

For this task, the participants were instructed to report what they perceived aurally or - in case of visual-only conditions - what they perceived visually (i.e. could read from the lips). Prior to each trial's stimulus presentation, a $1000 \mathrm{~ms}$ blank screen was presented followed by a jittering 600-800 ms black fixation cross on white background. After each trial, the three syllables (/ba/, /ga/, /da/) were displayed as response options (labeled 1, 2, 3), from which the answer had to be chosen and the respective number reported to the experimenter (three-alternative forced choice). As in SIFI, the next trial only started after the experimenter had entered the response.

Prior to the experiment, the intensity of the auditory stimulus delivered via headphones was set individually to the comfortable loudness level of each participant, which was adjusted in steps of $1 \mathrm{~dB}$ until it was judged as easily audible and medium loud, starting at $65 \mathrm{~dB}$ SPL. The mean medium loudness level of the auditory stimuli in the normal-hearing group was $66.9 \pm 2.6 \mathrm{~dB}$ SPL compared to $68.5 \pm 2.6 \mathrm{~dB}$ SPL in the hearingimpaired group.

\section{Sound-induced flash illusion (SIFI)}

235 In SIFI, visual flashes are paired with either a congruent or an incongruent number of auditory beeps, which may result in visual illusions when instructed to indicate the number of flashes perceived [27, 28].

Stimuli and set-up were similar to and described in more detail in [52], except for carrying out two runs of the test per participant, once with $0.5 \mathrm{kHz}$ beeps and once with $2 \mathrm{kHz}$ beeps in randomized order. We used these frequencies to investigate whether a low- versus a high frequency would affect the number of illusory perceptions, especially in the hearing-impaired group, whose hearing loss was characteristically present in the high-frequency range.

Each of the two runs consisted of seven conditions: Four unimodal conditions (one flash $1 F$, two flashes $2 F$, one beep $1 B$, two beeps $2 B$ ) as well as three bimodal conditions,

246 which comprised two congruent ones (one flash paired with one beep $1 F 1 B$, two flashes 247 paired with two beeps $2 F 2 B$ ) and the incongruent, illusory (or so-called fission) condition 
where one flash was paired with two beeps $(1 F 2 B)$. Unimodal and bimodal congruent conditions served as controls, while the incongruent one was used to quantify the strength of audiovisual integration in SIFI.

To determine the temporal binding window, eight stimulus onset asynchronies (SOAs: 70, 120, 170, 220, 270, 320, 370, $420 \mathrm{~ms}$ ) between the flashes and beeps were tested in conditions with multiple flashes or multiple beeps, with the first flash always starting simultaneously with the first beep. In the incongruent fission condition $(1 F 2 B)$, the second beep was always presented after the flash-beep pair. Each SOA was repeated ten times in bimodal $(1 F 2 B, 2 F 2 B)$ and five times in unimodal $(2 F, 2 B)$ conditions. Conditions without SOAs $(1 F, 1 B, 1 F 1 B)$ involved ten repetitions, resulting in 270 randomized trials in total for each run $(0.5 \mathrm{kHz}, 2 \mathrm{kHz})$. One run lasted about 15 minutes and was preceded by a short training session.

The participants were instructed to report the number of flashes they perceived and to ignore the beeps. Each trial started with a white fixation cross, centered on a black background, which the participants had to fixate throughout the whole trial. After 1000 $\mathrm{ms}$, the first flash was presented. After each trial, the subjects were asked to indicate the number of flashes to the experimenter, who entered the response before the next trial started. The two unimodal auditory control conditions $(1 B, 2 B)$ were tested in an immediately following, separate block, as in that case the instruction was to report the number of beeps heard.

As in the McGurk task, auditory stimuli were presented via headphones and the loudness adjusted analogously in steps of $1 \mathrm{~dB}$ to medium loudness for each participant prior the test and training session, starting at $70 \mathrm{~dB}$ SPL for the respective beep. The mean loudness level set in the normal-hearing group was $68.8 \pm 3.7 \mathrm{~dB}$ SPL for the $500 \mathrm{~Hz}$ beep and $69.4 \pm 3.8 \mathrm{~dB}$ SPL for $2 \mathrm{kHz}$, and $70.9 \pm 3.5 \mathrm{~dB}$ SPL and $71.5 \pm 3.9 \mathrm{~dB}$ SPL, respectively, in the hearing-impaired group.

\section{Audiovisual speech intelligibility-in-noise task}

\section{Audiovisual Oldenburg Sentence Test (AV-OLSA)}

276 One aim of the study was to link audiovisual integration capacities to audiovisual speech intelligibility in noise. Therefore, we used a newly developed audiovisual speech 
reception threshold (SRT) test: the Audiovisual Oldenburg Sentence Test (AV-OLSA) [53, see also 58,59$]$.

The AV-OLSA is based on the auditory-only female Oldenburg Sentence Test (Oldenburger Satztest, OLSA), a well-validated auditory SRT test with high precision [6063]. Llorach and colleagues developed a corresponding audiovisual version by recording video material with the original female speaker and "dubbing" that onto the original, validated auditory stimulus material. Just like the OLSA, the AV-OLSA is a matrix test using sentences of the form "name-verb-numeral-adjective-noun", with the array of words being a random combination from an inventory of 50 words.

In the AV-OLSA, participants heard these five-word sentences in background noise and simultaneously saw the speaker's head including her lip movement. After each trial, they repeated what they heard and the speech level then changed adaptively to obtain $80 \%$ SRTs. In the auditory-only (AO) condition, the auditory sentences were presented with a black screen.

We tested auditory-only (AO) and audiovisual (AV) conditions of 20 sentences each in fluctuating background noise (ICRA4-250). The ICRA4-250 originates from the ICRA4 noise of the International Collegium for Rehabilitative Audiology, that is a female, speechmodulated, and fluctuating noise [64]. In the ICRA4-250 noise, the maximum 'speech pauses' in that noise were limited to $250 \mathrm{~ms}$ (Wagener et al., 2006). While the presentation of the conditions was randomized, the preceding training with ten sentences each had a fixed order $(\mathrm{AO}, \mathrm{AV})$. The sentences were automatically chosen based on the previously tested lists, to avoid repetition.

In this experiment, we applied an open response format, i.e. after each sentence, the participant repeated all the words he or she has understood to the experimenter and was not given visual response alternatives like in a closed format. For each condition, the corresponding 80\%-SRTS were determined, referring to the signal-to-noise ratio (SNR) that yields $80 \%$ intelligibility based on word scoring. To obtain the threshold, an adaptive procedure [cf. 65] was applied; with a fixed background noise presentation level at $65 \mathrm{~dB}$ SPL and the target speech adaptively varying based on the previous answer, starting at 0 dB SNR. 


\section{Experimental procedure}

309 The study comprised three tests - SIFI, McGurk, AV-OLSA - performed in random order 310 across participants. SIFI and McGurk took place in a light- and sound-attenuated cabin, 311 where participants were seated approximately $60 \mathrm{~cm}$ in front of the screen. The 312 experimenter sat perpendicular to the set-up and, after each response, made the data 313 entry on a keyboard. In both tests, visual stimuli were presented on a 17" Iiyama ProLite 314 T 1731 SR-B1 monitor with a spatial resolution of $1280 \times 1024$ pixels and a luminance of $315200 \mathrm{~cd} / \mathrm{m}^{2}$ at a refresh rate of $60 \mathrm{~Hz}$. Sound stimuli were presented via Sennheiser 316 HDA200 headphones delivered by the USB audio interface RME Fireface UC 317 (http://www.rme-audio.de/en/products/fireface_uc.php). Stimulus presentation was 318 controlled by Presentation ${ }^{\circledR}$ software (Version 18.2, Neurobehavioral Systems, Inc., 319 Berkeley, CA, www.neurobs.com) run on an ASUS P8 Z77V computer equipped with an 320 Intel Core i7 950 processor and an ATI Radeon HD 5700 graphics card. Prior 321 measurements with an optical sensor and audio software confirmed synchronicity 322 between the visual and auditory stimuli and the correct timing of SOAs.

The AV-OLSA was completed in a sound-attenuated, quasi free-field room with the experimenter sitting laterally facing the participant with a different screen for making the data entry after each response. The loudspeaker (Genelec 8030A), delivering both speech and noise, was placed approximately $175 \mathrm{~cm}$ ahead in front of the participant $\left(\mathrm{S}_{0} \mathrm{~N}_{0}\right)$. The visual stimuli were presented on a 22" View Sonic TD 2220 Full HD monitor with a spatial resolution of $1920 \times 1018$ pixels, approximately $60 \mathrm{~cm}$ in front of the participant. Sound stimuli were delivered by the USB audio interface RME Fireface. The experiment was run on an ASUS Prime B250-PRO computer, equipped with an I5 $3.2 \mathrm{GHz}$ processor (generation 6), 16 GB RAM, and an on-board Intel HD S30 graphic card. Stimulus presentation was controlled by Matlab with the use of the VLC player for sentence and picture presentation, and Soundmex for background noise.

\section{Data analysis}

\section{Tests of audiovisual integration}

336 For both SIFI and McGurk, we first calculated each participant's proportion of correct 337 responses for each condition and SOA, as well as the proportion of illusory reports for 
each SOA in the respective incongruent, illusory conditions, i.e. $1 F 2 B$ for SIFI and $A V_{\text {incong }}$ for the McGurk task. Additionally, the mean values across SOAs were obtained for each individual.

Potential group differences between normal-hearing (NH) and hearing-impaired (HI) individuals were tested by means of repeated-measures general linear models (GLM), with Greenhouse-Geisser correction when the assumption of sphericity was violated. In those cases, adjusted $p$ values are reported and the deviation from sphericity indicated by epsilon ( $\mathrm{GG}_{\mathrm{eps}}$ ). To maintain a significance level of 0.05 in multiple pairwise comparisons or $t$ tests, Bonferroni correction was applied, and adjusted $p$ values are indicated.

\section{McGurk}

In a first analysis, we investigated the control conditions ( $A_{\text {only, }}, V_{\text {only, }}$ mean $A V_{\text {cong }}$ across SOAs) using a repeated-measures GLM on the proportion of correctly identified syllables with condition as within-subject factor (using the mean value across SOAs) and group $(\mathrm{NH}, \mathrm{HI})$ as between-subject factor.

The incongruent condition $A V_{\text {incong }}$ was analyzed separately to investigate possible group differences in the degree of susceptibility to the McGurk effect (defined as fusion reports /da/) for each of the nine SOAs (0-420 ms). Therefore, we first assessed the fusion reports by a repeated-measures GLM with SOA as within- and group as between-subject factor. In $A V_{\text {incong, }}$ responses can be classified as the auditory percept /ba/, the visual percept /ga/, or the (illusory) fusion percept/da/, indicating an integration of the visual and auditory syllable. To examine the response behavior in more detail, the respective response proportions across SOAs were submitted to a repeated-measures GLM with group as between-subject factor and response to the incongruent /ba-gav/ condition (/ba/, /ga/, /da/) as within-subject factor.

In addition, we wanted to quantify the temporal binding window for the McGurk task following a similar approach as in SIFI [cf. 52, 66]. When analyzing the fusion response to the incongruent $A V_{\text {incong }}$ condition, it should be kept in mind that some of those fusion responses could be attributed to errors in the percept of the unimodal components (i.e. auditory or visual percept) instead of reflecting true bimodal perception. Especially visual-only conditions can be prone to errors. Assume that a participant with a fusion response (/daAv/) of $60 \%$ for audiovisual, incongruent /baA-gav/ conditions might also 
perceive a visual /gav/ as /dav/ in $20 \%$ of the cases (visual error) and an auditory /baA/ as $/ \mathrm{da}_{\mathrm{A}} /$ in $3 \%$ of the cases (auditory error). These unimodal error rates have to be taken

371 into account to determine the number of "truly" audiovisually fused illusion responses [cf.

$37230,34]$. Hence, analogously to SIFI, we compared the proportion of fusion responses in $373 \mathrm{AV}$ incong to the proportion of visual and auditory errors in /gav/ and / $\mathrm{baA}_{\mathrm{A}} / \mathrm{trials}$, 374 respectively, using Bonferroni-corrected paired-sample $t$ tests. The contiguous span of successive SOAs in which the fusion proportion was significantly higher than the combined error proportion then determined the temporal limits of the window of integration for the McGurk task [cf. 52, 66].

\section{SIFI}

379

380

As in the McGurk task, we first investigated proportion of correct responses in the control conditions $(1 F, 2 F, 1 B, 2 B, 1 F 1 B, 2 F 2 B)$ of each frequency by a repeated-measures GLM with frequency and condition as within-subject factors (using the mean value across SOAs) and group (NH, HI) as between-subject factor.

Secondly, we probed whether the groups showed an overall susceptibility to the illusion by accounting for errors in the congruent condition. To that end, we compared the proportion of illusory responses (i.e. more than one flash) in the incongruent fission condition $(1 F 2 B)$ to the congruent control condition $(1 F 1 B)$, again using a repeatedmeasures GLM for each frequency with condition (mean across SOAs) and group (NH, HI) as within- and between-subject factor, respectively.

For the investigation of potential group differences in the amount of illusion across SOAs, a repeated-measures GLM on the proportion of illusory responses in the $1 F 2 B$ condition for each SOA was applied, with SOA (70-420) and frequency $(0.5 \mathrm{kHz}, 2 \mathrm{kHz})$ as within- as well as group (NH, $\mathrm{HI})$ as between-subject factor.

Finally, to determine the temporal binding window in SIFI for each group, the same approach as in [52] was applied, that is, the temporal limit of the illusion was defined as the contiguous span of consecutive SOAs in the $1 F 2 B$ condition in which the proportion of illusory reports was significantly higher than in the $1 F 1 B$ control condition, as determined by Bonferroni-corrected paired-sample $t$ tests [cf. 66]. This was carried out for each frequency separately. 
400 To investigate the relationship between the two tests in each group, we calculated 401 Pearson's correlation scores between the mean fusion responses in the McGurk and the 402 mean illusory responses in SIFI (mean across SOAs and frequencies) for both the HI and 403 NH groups.

\section{Test of audiovisual speech intelligibility in noise (AV-OLSA)}

405 From the AV-OLSA, we obtained one 80\%-SRT value for both auditory-only (AO) and audiovisual (AV) condition, referred to as AO-SRT 80 and AV-SRT80. To assess the audiovisual gain, we further calculated the difference between both intelligibility scores, i.e. AV-SRT 80 - AO-SRT80.

409 Relation of audiovisual integration and audiovisual speech 410 intelligibility in noise

411 Finally, we linked audiovisual integration to audiovisual speech-in-noise recognition. As 412 we were interested in whether, and to what extent, audiovisual integration capacities can 413 explain variability in audiovisual speech intelligibility in noise beyond age and hearing 414 abilities, we performed a stepwise linear regression for each group with $A V$-SRT80 as 415 outcome variable and Age, PTA-4, Mean \% SIFI illusion (mean across SOAs and mean 416 across frequencies due to correlation), as well as Mean \% McGurk illusion (mean across 417 SOAs) as potential predictor variables.

418 The stepwise approach used both an inclusion and removal criterion for variable 419 selection. At each step of the regression, one variable of the set of potential predictor 420 variables that has not yet been entered and has the smallest probability of $\mathrm{F}$ is added into 421 the regression equation if that probability is sufficiently small (0.05). Simultaneously, 422 variables already in the equation are eliminated if their F probability becomes sufficiently 423 large (0.10). The approach stops at a model where no more variables are suitable for 424 inclusion or removal.

425 All statistical analyses were performed with IBM SPSS Statistics for Windows, version 426 24.0 . 


\section{Tests of audiovisual integration}

429

430

432

433

434

435

436

437

438

439

440

\section{McGurk}

We first investigated the control conditions ( $A_{\text {only }}, V_{\text {only, mean }} A V_{\text {cong }}$ across SOAs) to compare the correct identification of syllables between groups (see Fig 2). While the main effect of condition was found to be significant $\left[F_{(2,96)}=72.73, p_{a d j}<0.001, \mathrm{GG}_{\mathrm{eps}}=0.62\right.$, $\left.\eta^{2}=0.60\right]$, the main effect of group was not $\left[F_{(1,48)}=2.24, p=0.141\right]$. Post-hoc comparisons did not show a significant improvement from auditory-only to audiovisual congruent conditions across both groups $\left[\mathrm{d}_{\mathrm{AV}-\mathrm{A}}=2.2, \mathrm{~T}_{(49)}=1.61, p_{\text {adj }}=0.114\right]$. The benefit from both audiovisual and auditory-only presentation over visual-only conditions, however, was significant $\left[\mathrm{d}_{\mathrm{AV}-\mathrm{V}}=27.0, \mathrm{~T}_{(49)}=9.82, p_{a d j}<0.001 ; \mathrm{d}_{\mathrm{A}-\mathrm{V}}=24.8, \mathrm{~T}_{(49)}=7.18, p_{a d j}<0.001\right]$.

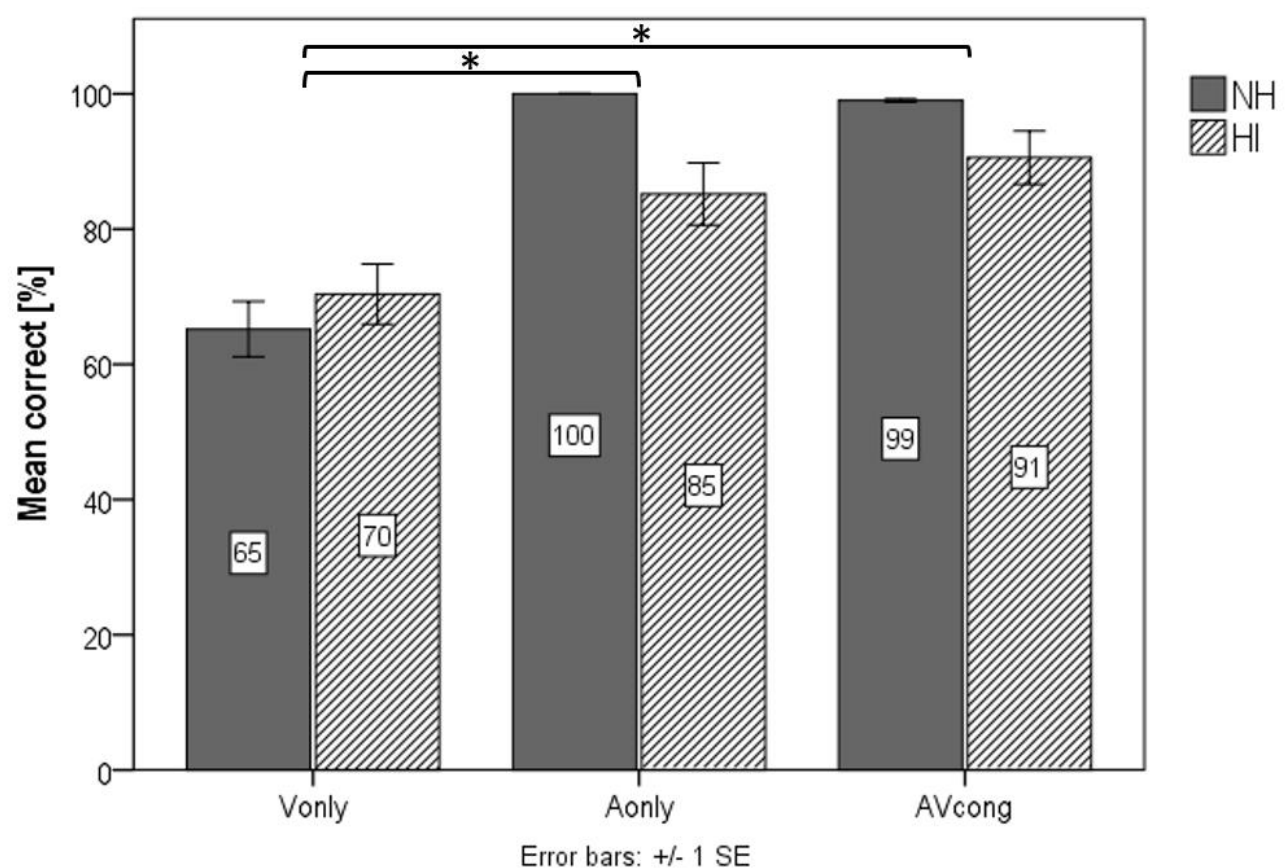

Fig 2: Mean percent correct responses (across SOAs) in each control condition

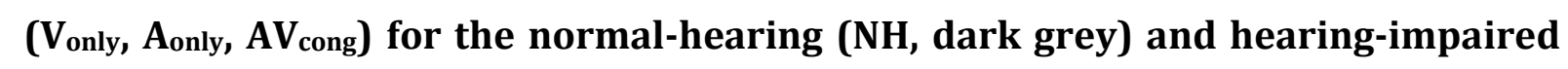
(HI, striped) groups. Asterisks indicate significant differences between conditions (both $p_{a d j}<0.001$ ) in post-hoc comparisons (mean across groups) following a significant main effect of condition in the corresponding ANOVA $\left(p_{a d j}<0.001\right)$. 
To investigate potential group differences in the susceptibility to the McGurk effect, 446 the proportion of fusion reports (/da/) in the audiovisual incongruent condition $A V_{\text {incong }}$ 447 were analyzed for each SOA, exposing a significant main effect of group with a large effect 448 size $\left[F_{(1,48)}=18.35, p_{a d j}<0.001, \eta^{2}{ }_{p}=0.23\right]$. Overall across all SOAs, HI individuals showed a 449 higher number of fusion reports and thus, a higher susceptibility to the McGurk effect, 450 than normal-hearing individuals ( $42 \pm 30 \%$ vs. $11 \pm 20 \%$ ), consistent with hypothesis H1. 451 Both main effect of SOA and SOA $x$ group interaction likewise yielded significance [SOA: $452 F_{(8,384)}=14.96, p_{a d j}<0.001, \eta^{2}{ }_{p}=0.24$; interaction: $\left.F_{(8,384)}=4.38, p_{a d j}=0.002, \eta^{2} \mathrm{p}=0.08\right]$ with 453 decreasing illusory reports with increasing SOAs in the HI group.

Since in $A V_{\text {incong }}$ conditions participants could either report the illusory fusion percept 455 /da/ by integrating both presented syllables, or respond in line with the auditory (/ba/) 456 or the visual (/ga/) component, we examined the response behavior in more detail. This 457 resulted in a significant main effect of response $\left[F_{(2,96)}=50.44, p_{a d j}<0.001, \eta^{2}{ }_{p}=0.51\right]$, but 458 also a significant response $x$ group interaction $\left[F(2,96)=19.80, p_{a d j}<0.001, \eta^{2} p=0.29\right]$. Post459 hoc analysis revealed that across all SOAs, HI individuals reported the fusion percept 460 significantly more often than NH individuals [42 $\pm 30 \%$ vs. $11 \pm 20 \%$, $\mathrm{d}_{\mathrm{HI}-\mathrm{NH}}=30.7$, $\left.461 \mathrm{~T}_{(48)}=4.28, \mathrm{p}_{a d j}<0.001\right]$, whereas $\mathrm{NH}$ answered more frequently in line with the auditory 462 component of the presentation, i.e. gave the 'correct' response /ba/, compared to HI [86 $463 \pm 25 \%$ vs. $\left.45 \pm 33 \%, \mathrm{~d}_{\mathrm{HI}-\mathrm{NH}}=-40.5, \mathrm{~T}_{(48)}=-4.89, p_{a d j}<0.001\right]$. At the same time, HI also 464 responded more often in line with the visual component (/ga/), i.e. showed a higher visual 465 bias than $\mathrm{NH}\left[13 \pm 19 \%\right.$ vs. $\left.3 \pm 6 \%, \mathrm{~d}_{\mathrm{HI}-\mathrm{NH}}=9.79, \mathrm{~T}_{(48)}=2.48, p_{a d j}=0.017\right]$. Fig 3 shows the 466 response behavior for the two groups across SOAs. 


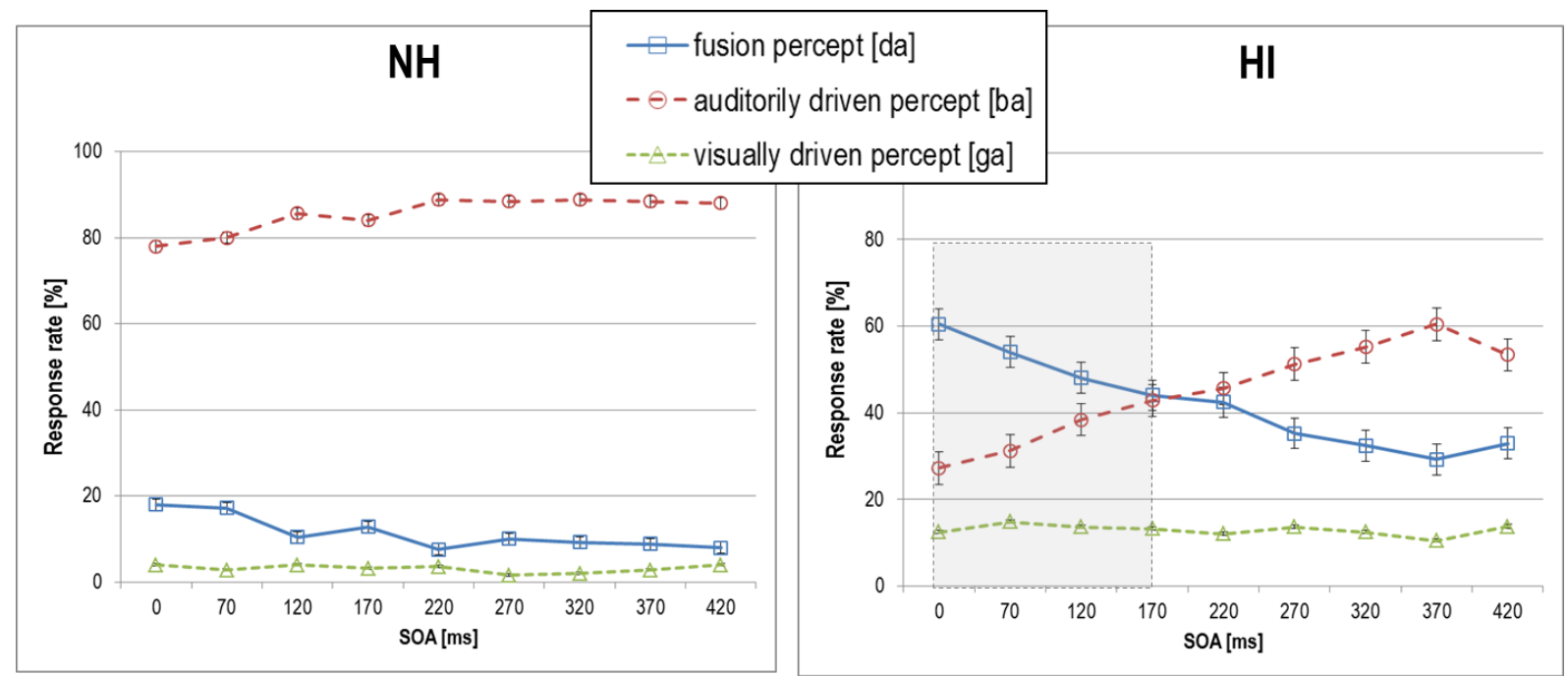

Fig 3: Response rate across SOAs in the incongruent audiovisual /baA-gav/ condition ( $\mathrm{AV}_{\text {incong) }}$ of the McGurk task for the normal-hearing ( $\mathrm{NH}$, left panel) and hearing-impaired (HI, right panel) group. Possible responses were the auditorilydriven percept [ba], the visually-driven percept [ga] or the fusion percept [da] indicating integration of the auditory and visual syllable. The shaded area reflects the window of integration for the HI group. NH individuals relied largely on the auditory percept, whereas the HI group reported significantly more illusory fusion responses. HI individuals were most likely to integrate visual and auditory syllables in asynchronies up to $170 \mathrm{~ms}$ between the lip movement and the spoken syllable. With increasing SOAs, the rate of fusion responses decreased, while the auditory response rate increased, intersecting at $170 \mathrm{~ms}$.

Furthermore, we aimed at obtaining the temporal binding window for the McGurk task in each group, accounting for unisensory visual and auditory errors in the audiovisual fusion percept. Comparing the illusory /da/ reports in the incongruent condition to /da/ reports in visual-only /gav/ and auditory-only /baA/ trials (error proportions) for each SOA resulted in significant 'true' fusion percepts ranging from 0 to $170 \mathrm{~ms}$ in the HI group (all $p_{a d j}<0.05$ ), while the temporal window could not be determined for NH not showing the illusion. The window coincides with the intercept, where the number of auditorilydriven responses exceeds the number of fusion responses (Fig 3). an audiovisual illusion in an auditory task, with the highest probability of illusory 
percepts in asynchronies up to $170 \mathrm{~ms}$ between the lip movement and the spoken syllable.

491 Outside of this window of $170 \mathrm{~ms}$, they responded more in line with the 'correct' auditory percept. However, some individuals of the HI group also relied on the visual percept. The

493 NH individuals were significantly less prone to the McGurk illusion, but responded in line 494 with the correct auditory component. Compared to the NH group, the HI group showed 495 significantly more fusion responses across all SOAs. Furthermore, across both groups, increasing hearing loss was associated with increased audiovisual integration and reduced auditory bias.

498

\section{SIFI}

The analysis of the control conditions $(1 F, 2 F, 1 B, 2 B, 1 F 1 B, 2 F 2 B)$ across both frequencies $(0.5 \mathrm{kHz}, 2 \mathrm{kHz})$ and between the two groups ( $\mathrm{NH}, \mathrm{HI})$ exposed a significant main effect of condition $\left[F_{(5,235)}=82.59, p_{\text {adj }}<0.001, G_{\text {eps }}=0.54, \eta^{2}=0.64\right]$. At the same time, neither the main effect of group nor the main effect of frequency yielded significance, with $F_{(1,48)}=0.222, p=0.64$ and $F_{(1,48)}=0.001, p=0.98$, respectively. Likewise, none of the two-way or three-way interactions between condition, frequency, and group were significant, indicating that the performance of both groups was similar across the control conditions, independent of the frequency. To follow up on the main effect of condition, post-hoc multiple comparisons were performed and revealed that conditions with a single flash/beep $(1 F, 1 B, 1 F 1 B)$ did not differ from each other, but did significantly differ from conditions with multiple flashes/beeps $(2 F, 2 B, 2 F 2 B)$, which in turn also significantly differed amongst each other (all $p_{a d j}<0.05$ ). For both frequencies, both $\mathrm{NH}$ and $\mathrm{HI}$ experienced a benefit from congruent bimodal presentations $(2 F 2 B)$ as compared to the unimodal visual condition $(2 F)$, but performed best in the unimodal auditory $2 B$ condition (across all SOAs).

To test whether groups showed an overall susceptibility to the illusion, we compared the proportion of multiple-flash reports in the incongruent illusory $1 F 2 B$ condition (collapsed across SOAs) to the $1 F 1 B$ control condition. While significantly more illusory reports were observed in the incongruent than in the control condition [main effect of condition $\left.F_{(1,48)}=22.5, p<.001, \eta^{2}{ }_{p}=0.32\right]$, groups did not differ significantly from each other [main effect of group $\left.F_{(1,48)}=0.009, p=0.925\right]$. Apparently, both $\mathrm{NH}$ and HI individuals experienced the illusion similarly when averaging across SOAs, with no notable group differences, thus not supporting $\mathrm{H} 1$. 
To further analyze the susceptibility to the flash illusion in the $1 F 2 B$ condition in more

524 detail by taking into account the varying SOAs, the number of illusory reports for each 525 SOA was analyzed. Here, we found an expected significant main effect of SOA $526\left[F_{(7,329)}=29.38, p_{a d j}<0.001, \quad G_{\text {eps }}=0.71, \quad \eta^{2}{ }_{p}=0.39\right]$, with post-hoc tests confirming 527 decreasing numbers of illusion with increasing SOAs. Again, the main effect of group was 528 not significant $\left[F_{(1,48)}=0.011, p=0.915\right]$, nor was the main effect of frequency $\left[F_{(1,48)}=0.125\right.$, $p=0.725]$, or any of the interactions between SOA, frequency, and group. This suggests that $\mathrm{NH}$ and $\mathrm{HI}$ did not differ from each other in their illusory reports, but both experienced fewer illusions with increasing SOAs, similarly in both frequencies $0.5 \mathrm{kHz}$,

$5322 \mathrm{kHz}$ ). A paired sample $t$ test conducted groupwise between the frequencies for each SOA 533 revealed no significant intra-group difference between the frequencies when corrected 534 for multiple comparisons.

Finally, to determine the frequency-specific temporal binding window for each group, we applied the approach by Foss-Feig and colleagues $[52,66]$. For the $0.5 \mathrm{kHz}$ beep, we 537 found that both NH and HI groups showed significantly higher illusory reports in the $1 F 2 B$ 538 compared to the $1 F 1 B$ condition successively across SOAs of 70-120 ms (all $p_{a d j}<0.05$ ), 539 which defines the temporal limits of the integration window. In the $2 \mathrm{kHz} \mathrm{run}, \mathrm{HI}$ 540 individuals showed the same window of integration $(-120 \mathrm{~ms})$, whereas the NH group's 541 window was extended by one SOA $(-170 \mathrm{~ms})$ (all $\left.p_{a d j}<0.05\right)$. These results, as shown in 542 Fig 4, indicate a slightly broader window in $\mathrm{NH}$, but only for the high-frequency tone.
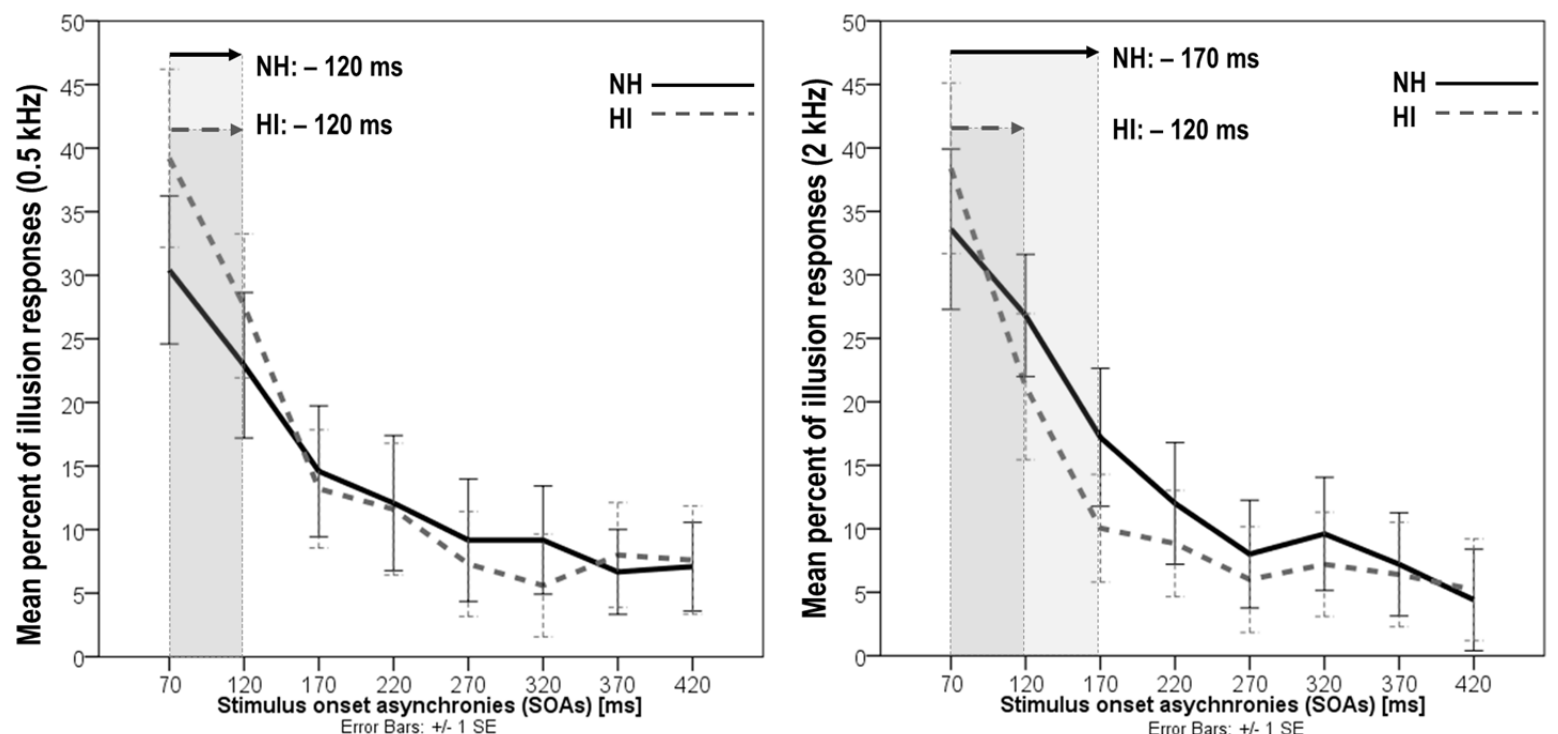

544 Fig 4: Percent of illusion in the incongruent 1F2B condition of SIFI. Shown are the

545 'two flashes' reports across SOAs for the normal-hearing ( $\mathrm{NH}$, solid lines) and hearing- 
546 impaired (HI, dashed lines) group in the $0.5 \mathrm{kHz}$ run (left) and the $2 \mathrm{kHz}$ run (right). The

547 shaded areas show the respective temporal binding window for each group.

\section{Correlation between SIFI and McGurk}

550 To investigate the relationship between the two tests, we calculated correlation scores, 551 but did not find a significant relationship in either group [HI: $r=-0.110, p=.599$; NH: $r=-$ $5520.023, p=0.913)$.

\section{Test of audiovisual speech intelligibility in noise (AV-OLSA)}

554 To test speech-in-noise intelligibility, we applied the audiovisual SRT test AV-OLSA, 555 obtaining 80\%-SRTs in fluctuating background noise for the auditory-only (AO) and 556 audiovisual (AV) conditions. Fig 5 shows the boxplots for each condition as well as the 557 gain from adding visual information, defined as SRT difference between AV and AO 558 conditions. As hypothesized in H2, normal-hearing individuals showed significantly 559 lower, i.e. better SRTs in both the AO condition compared to the HI group [-8.3 dB SNR \pm 5602.1 vs. $-0.8 \mathrm{~dB}$ SNR $\left.\pm 4.1, \mathrm{~d}_{\mathrm{NH}-\mathrm{HI}}=-7.5 \mathrm{~dB}, T_{(48)}=-8.12, p_{\mathrm{adj}}<0.001\right]$ and in the $\mathrm{AV}$ condition $561 \quad\left[-15.4 \mathrm{~dB}\right.$ SNR \pm 4.9 vs. $-7.5 \mathrm{~dB}$ SNR $\left.\pm 3.7, \mathrm{~d}_{\mathrm{NH}-\mathrm{HI}}=-7.9 \mathrm{~dB}, T_{(48)}=-6.41, p_{\text {adj }}<0.001\right]$. This 562 means that the signal-to-noise ratio could be much lower for NH individuals in order to 563 understand $\sim 80 \%$ of the words.

564 Contrary to H2, however, the gain from adding visual information was almost equal 565 in both groups, with $-7.1 \mathrm{~dB} \pm 4.2$ for $\mathrm{NH}$ and $-6.7 \mathrm{~dB} \pm 3.3$ for $\mathrm{HI}$ participants [d $\mathrm{NH}-\mathrm{HI}=-$ $\left.5660.4 \mathrm{~dB}, T_{(48)}=-0.34, p=0.74\right]$. Apparently, both groups benefitted equally from adding 567 visual information. Note, however, the larger variability among the NH participants in the 568 AV condition and in the gain. 

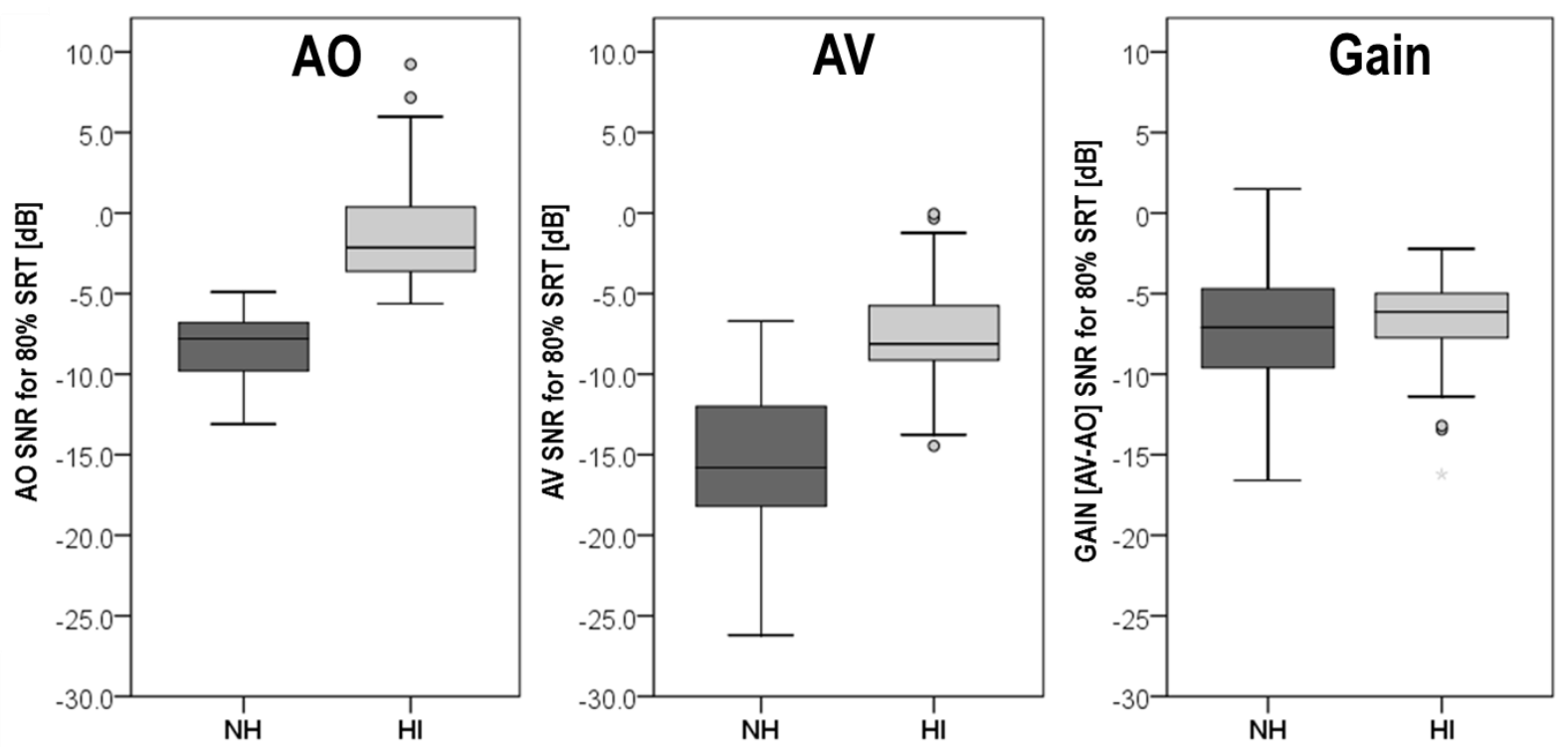

Fig 5: 80\%-SRTs for auditory-only (AO, left) and audiovisual (AV, middle) conditions as well as the gain (AV-AO, right) for the normal-hearing ( $\mathrm{NH}$, dark grey) and hearing-impaired (HI, light grey) participants. The speech-reception thresholds indicate the signal-to-noise ratios where $\sim 80 \%$ of the speech is understood. $\mathrm{NH}$ individuals showed lower, i.e. better, SRTs than in both auditory-only and audiovisual conditions, whereas the gain obtained from additional visual information was almost equal in both groups.

\section{Relation of audiovisual integration and audiovisual speech} recognition in noise

In the last step, we linked audiovisual integration capacities, as determined by the susceptibility to the flash- and McGurk illusion, to audiovisual speech-in-noise intelliibility, to explore whether, and how much variance in audiovisual SiN recognition (AV-SRT $\left.{ }_{80}\right)$ can be explained by AV integration variables - beyond age and hearing loss.

To that end, a stepwise linear regression was performed on the AV-SRT 80 with Age, PTA-4, Mean \% SIFI illusion (across both frequencies) and Mean \% McGurk illusion as predictor variables.

Table 1 shows the final model for each group with the selected variables in respective order of selection, as well as the change in variance explained (adjusted $\mathrm{R}^{2}$ ). For $\mathrm{NH}$ participants, Age and McGurk illusion account together for 29\% overall variance in 
590

591

592

593

594

595

audiovisual speech intelligibility, of which $12 \%$ is explained by the McGurk illusion. In HI individuals, more overall variance of speech intelligibility is explained (60\%) by PTA-4 and both tests of AV integration (SIFI, McGurk), with the latter accounting for $25.5 \%$. The signs of the beta coefficients indicate that those with more McGurk illusions and fewer SIFI illusions have better (i.e. lower) audiovisual SRTs. Overall, these results support hypothesis $\mathrm{H} 3$.

Table 1: Prediction of audiovisual speech intelligibility (AV-SRT80) by age, hearing loss, and tests of audiovisual integration for normal-hearing (NH) and hearingimpaired (HI) participants.

\begin{tabular}{cclccccc}
\hline Group & Step & Predictor included & Beta & $\mathrm{p}$ & $\mathrm{R}^{2}$ & $\mathrm{R}^{2}{ }_{\mathrm{adj}}$ & $\begin{array}{c}\mathrm{R}^{2}{ }_{\mathrm{adj}} \\
\text { change }\end{array}$ \\
\hline $\mathrm{NH}$ & 1 & Age & .642 & .004 & .207 & .171 & \\
& 2 & Mean \% McGurk illusion & -.423 & .042 & .352 & .290 & .119 \\
\hline $\mathrm{HI}$ & 1 & PTA-4 & .678 & .000 & .372 & .345 & \\
& 2 & Mean \% Flash illusion & .420 & .004 & .564 & .525 & .180 \\
& 3 & Mean \% McGurk illusion & -.305 & .034 & .650 & .600 & .075 \\
\hline
\end{tabular}

Shown are the predictors entering the final model of the stepwise linear regression for predicting AV speech intelligibility (80\%-SRT in AV conditions) with the corresponding beta coefficients as well as the order of variable selection. Furthermore, the corresponding change $\left(\Delta \mathrm{R}^{2}\right.$ adj $)$ in the amount of the adjusted coefficient of determination $\left(\mathrm{R}^{2}\right.$ adj) is presented, reflecting the proportion of explained variance by adding the predictor to the regression model.

\section{Discussion}

In this study, we investigated the influence of age-related, untreated, mild hearing impairment on audiovisual integration capacities and linked those for the first time to audiovisual speech-in-noise recognition to evaluate the amount of (additional) predictive value beyond age and hearing loss.

We hypothesized that hearing loss would exert an influence on audiovisual processing such that H1) groups would differ in their AVI capacities, and H2) groups 
612 would differ in the audiovisual SiN recognition as well as in the gain obtained from visual 613 information. Moreover, we probed H3) whether, and to what extent, audiovisual SiN 614 intelligibility can be predicted by measures of AVI, expecting this to be particularly the 615 case for the HI group and the McGurk task using speech stimuli.

With regard to hypothesis H1), results indicated that groups differed in their AVI 617 capacities and the corresponding window of integration, yet only indicated by the McGurk 618 illusion with HI participants showing increased audiovisual integration compared to the 619 NH group. As to hypothesis H2), the HI group showed expectedly worse SRTs in audioonly and audiovisual SiN conditions, yet did not differ from $\mathrm{NH}$ in their audiovisual gain.

621 Concerning hypothesis H3), AVI capacities indeed accounted for additional variability in 622 audiovisual SiN recognition beyond age and hearing loss, especially in the HI group.

\section{Audiovisual integration capacities}

\section{McGurk}

625 The McGurk illusion is an established paradigm to assess audiovisual integration on a behavioral level $[26,67]$. In contrast to SIFI using low-level stimuli, the McGurk illusion uses speech syllables and can thus be regarded as a gauge particularly for audiovisual speech integration. Aiming at assessing the influence of mild hearing loss on these measures, we compared the strength of AV speech integration capacities between $\mathrm{NH}$ and HI participants using the susceptibility to the illusion as an indicator.

As hypothesized, elderly HI individuals were significantly more prone to the McGurk 632 illusion, indicating greater AV speech integration capacities compared to their NH peers. 633 The effect of mild hearing impairment on AVI was evident across all SOAs ranging from 0 $634 \mathrm{~ms}$ (synchronous presentation) up to $420 \mathrm{~ms}$, with the auditory stimulus presentation 635 lagging the visual one, as groups differed significantly across all ranges. Compared to the 636 HI group, the NH individuals showed less AV speech integration and responded most 637 often in line with the (correct) auditory component. At the same time, both fusion and 638 (correct) auditory responses correlated positively and negatively, respectively, with the 639 degree of hearing loss. multisensory integration abilities [e.g., 36-40, 42-44, 68, 69, for reviews see 45, 46], the 
results on effects of age-related hearing impairment on AVI are comparatively scarce, 643 especially for mild-to-moderate hearing loss. This is aggravated by the fact that studies 644 use different tests and measures of AVI, which might yield different results; this 645 emphasizes the importance of interpreting results in a task-specific context [cf. 70].

We found only two studies comparing AVI capacities by means of the McGurk illusion in untreated mild-to-moderate hearing loss. First, our findings replicated results of Rosemann and Thiel [49], who found enhanced AVI capacities in mild-to-moderately HI compared to $\mathrm{NH}$ individuals as indicated by McGurk fusion percepts, and who likewise reported a significant correlation between response behavior and hearing loss. Note that we also used a subset of their stimuli. Similarly, our results are in line with those of Stropahl and Debener [71], who investigated mild-to-moderately hearing impaired and normal-hearing subjects as well as cochlear-implant (CI) patients. They reported increased McGurk illusions in the HI compared to the NH group, with the latter more frequently choosing the auditory response, whereas the CI patients did not display significantly higher illusion rates compared to $\mathrm{NH}$, but rather responded in line with the visual component of the McGurk condition.

We extended those findings by additionally varying the SOAs, thus assessing the corresponding temporal window of (speech) integration, i.e. the time frame in which auditory and visual speech syllables are most likely to be integrated while at the same time reflecting a true bimodal response rather than unimodal errors [cf. 30]. While for the

662 NH group, the window could not be determined with our approach due to overall low 663 fusion reports, the HI group experienced expectedly fewer illusions with increasing SOAs 664 and showed a temporal window of speech integration up to $170 \mathrm{~ms}$, with auditory 665 information lagging visual. Thus, when auditory and visual speech stimuli were presented 666 within this window, the probability of AV speech integration for hearing-impaired 667 individuals was highest. With increasing temporal disparities beyond that window, the HI 668 group was rather driven by their auditory perception. Some individuals, however, also relied on the visual percept.

Some studies have investigated the influence of temporal disparity between the 671 stimuli in the McGurk effect in (younger) healthy adults [e.g., 30, 72, 73], consensually 672 suggesting the illusion to be relatively robust against higher latencies of the auditory 673 information. Although our results seem to match those of Van Wassenhove [30] and 
674 Munhall and colleagues [73], reporting significant fusion responses up to $+170 \mathrm{~ms}$ and $675+180$ ms (audio lag), respectively, we cannot directly compare the window, as they 676 measured undergraduate students and used a different approach to determine the limits 677 of the window. At the same time, the window width depends on the task, stimuli, 678 individual factors, training, and approaches of quantification [e.g., 31, 34, 74-77]. Thus, 679 numerically, we can only compare our two groups suggesting that untreated hearing680 impaired individuals are more likely to integrate even asynchronous audiovisual speech than their normal-hearing peers in an auditory task.

Although our groups did not differ in the visual-only condition, the HI group showed 683 a small but significantly higher bias for the visual component in the incongruent McGurk 684 condition compared to NH individuals. This resembles the pattern in Stropahl and 685 Debener's study [71], although it was not reported by Rosemann and Thiel [49]. Other studies using the McGurk illusion in CI patients have similarly found them to show a bias towards visual information, presumably by placing more weight on the visual modality due to their degraded auditory modality [e.g., 71, 78-82, for review see 51].

Taken together, these findings can be interpreted in the light of theories that put 690 forward a weighting of information and modalities according to their reliability, which in 691 turn affects audiovisual integration [e.g., 83-85, for review see 86]. Accordingly, the more 692 reliable modality dominates audiovisual processing and overall perception. CI users with 693 profound hearing loss and hearing-impaired individuals without hearing aids experience 694 degraded auditory input, therefore they might place more weight on the visual modality 695 (relative to NH individuals) in an auditory task such as the McGurk. Hence, they either 696 integrate auditory and visual information more, as indexed by higher fusion reports 697 compared to NH listeners (this study, $[49,71]$, and/or display a higher bias towards the 698 visual component.

699 Summing up, our results for the McGurk task indicate that even mild, untreated 700 hearing impairment affects audiovisual speech integration, with increasing hearing loss 701 being associated with increased integration in an auditory task. At the same time, it 702 extends previous findings, in that we found the window of speech integration to comprise $703 \sim 170 \mathrm{~ms}$ for HI listeners. 
In contrast to the McGurk task, we did not find group differences in the strength of audiovisual integration as indexed by the susceptibility to the flash illusion, for neither low nor high frequency. This was contrary to our expectations of overall higher illusory reports in the $\mathrm{NH}$ group. In line with the above-stated model of cue weighting according to reliability, we hypothesized that the HI individuals would place more relative weight on the visual modality and thus experience fewer visual illusions. At the same time, we wanted to probe a possible interaction effect of frequency, as it was conceivable that HI experience more illusions in the $0.5 \mathrm{kHz}$ conditions, as this resembles a frequency range which should not be affected by the age-related, high-frequency loss. Unexpectedly, we

714 found that $\mathrm{HI}$ and $\mathrm{NH}$ individuals were equally prone to the visual illusion across SOAs 715 from 0 to $120 \mathrm{~ms}$, with neither inter-group nor intra-group differences for the frequency. 716 The only difference was found for the window size in the $2 \mathrm{kHz}$ run, where $\mathrm{NH}$ individuals showed a slightly broader window comprising $170 \mathrm{~ms}$.

Previous studies using the flash illusion with varying SOAs have reported temporal windows up to $150 \mathrm{~ms}$ in young, healthy adults $[28,69,87]$, compared to a greater susceptibility along with broader windows (up to SOAs of $400 \mathrm{~ms}$ ) in elderly, normal-

721 hearing adults $[69,87,88]$. In contrast, investigations in older adults with hearing 722 impairment using the flash illusion were nonexistent and just recently complemented by 723 a study of ours, in which we found differences between mildly hearing-impaired hearing724 aid users in contrast to those with the same degree of hearing loss who had not yet been 725 treated with hearing aids [52]. There, we found greater audiovisual integration together 726 with a wider window of integration for the treated compared to the untreated group of HI 727 subjects, with the former displaying an integration window of $370 \mathrm{~ms}$ compared to 220 $728 \mathrm{~ms}$ in the latter. We put those results in perspective with other studies and argued that 729 both hearing impairment and hearing-aid use may affect audiovisual integration, 730 speculating that the possibly adverse effects of hearing loss on audiovisual integration (as 731 indexed by SIFI) might be reversed by hearing-aid use. To shed light on the separate 732 effects of hearing loss and hearing-aid use, we stressed the need for a normal-hearing 733 control group, which we wanted to complement with this study. Although the untreated group of our previous study was comparable to our current 735 HI group with respect to age and hearing loss, and we used the same SOAs as well as the 
approach to window quantification [66], we did not obtain exactly the same window size

737 for the HI group, but a narrower one in this study. This might be due to (a) an overall 738 lower susceptibility in the current cohort compared to the old data (mean \% illusion 739 across SOAs $14 \%$ vs. 18\%) with a lower onset of susceptibility reports in the shortest SOA 740 of $70 \mathrm{~ms}$ (39\% vs. 44\%), and (b) an apparently sharper drop in illusion reports in the 741 current group in SOAs of up to $170 \mathrm{~ms}$, compared to the less sloping curve in the group of 742 the previous study. The differences in results might be explained by the overall intra- and inter-individual variability in the perception of the flash illusion $[89,90]$.

While we cannot conclusively explain the discrepancy between the previous and 745 current data for the untreated HI group, the actual comparison of interest here is between 746 the groups of untreated $\mathrm{HI}$ and NH individuals. We were interested in whether a mild 747 hearing loss would have an influence - independent from hearing-aid use - on audiovisual 748 integration in a visual task having an incongruent auditory input. We assumed NH 749 individuals to be more prone to the illusion, as they may place more weight on their intact, 750 reliable auditory modality relative to $\mathrm{HI}$ individuals and are thus, more influenced by the 751 incongruent number of beeps [cf. 52]. In contrast, we expected the HI individuals to show 752 a shift towards reliance on the visual modality, being less influenced by their degraded 753 auditory modality. This would have been indicated by more correct and fewer reports of 754 illusions in the HI compared to the NH group, and possibly also an interaction in the sense 755 that for the higher frequency this might be more apparent than in the unaffected low 756 frequency range. However, our current data does not support these hypotheses.

In contrast to the McGurk effect, SIFI represents a task requiring better temporal 758 discrimination abilities, which in turn is a key determinant for the weighting process 759 determining whether signals are integrated [34]. While the precision of temporal 760 processing abilities decline with age [38, 87, 91-94, for review see 45] and older adults 761 show a shift towards visual dominance, recent work indicates that the relation between 762 temporal processing and perceptual multisensory integration becomes decoupled in 763 (healthy) aging [70, 95, for review see 96]. Importantly, Fitzgibbons and Gordon-Salant 764 [93] found the impaired temporal acuity in older compared to younger adults to be 765 independent of hearing loss. Thus, compromised temporal processing, as also reflected by 766 a broader temporal binding window [34, 70, 76, 87, 97], might become apparent as an 767 effect of aging but not as a direct effect of (mild) age-related hearing loss. 
The finding that both $\mathrm{HI}$ and NH individuals weighted the auditory modality equally in a visual task requiring temporal discrimination supports the concept that the overall superior temporal discrimination ability of audition persists in mild hearing loss (or has

771 been recalibrated). Compared to the hearing-aid users of our previous study, however, both show decreased illusion reports indicating that they still rely more on the visual modality relative to hearing-aid users.

Summing up, both HI and NH participants are equally likely to integrate low-level audiovisual input in a visual task within a window of 120 (or $170 \mathrm{~ms}$, respectively), when the auditory information lags the visual. Group differences in the strength were not observed, thus not giving evidence for a separate effect of a mild hearing loss on audiovisual integration abilities for an auditory-modulated visual task requiring temporal processing with low-level stimuli.

\section{Relation between SIFI and McGurk}

We expected the direction of potential differences in audiovisual integration between the groups to be dependent on the specific test of audiovisual integration, yet did not detect a significant correlation between the two tests for either group.

Although both are classical paradigms for assessing audiovisual integration on a behavioral level, they are fundamentally different. In SIFI, visual perception is affected by incongruent auditory information, whereas in McGurk, auditory perception is altered by incongruent visual information. Moreover, SIFI uses low-level stimuli, whereas the McGurk task uses speech syllables. Despite these task differences, Stevenson and colleagues [34] found both to be negatively correlated in younger adults. Hence, those with more McGurk illusions showed a lower susceptibility to the flash illusion and vice versa. These findings are not supported by our results, indicating that the tests might reflect two different aspects of audiovisual processing. Support for this notion also comes from a study by Tremblay et al. [98], who used both tests in a developmental study in children, reporting the strength of audiovisual speech integration to vary with increasing development, whereas the non-speech illusion remained stable across ages.

A previous study has put forward the idea of - at least partly - different mechanisms underlying integration effects of speech compared to non-speech material [99], supported by electrophysiological studies [100-102]. A review by Calvert [35] concluded that 
distinct crossmodal interactions also involve distinct brain areas that are differently involved in multisensory integration processes [see also 103]. She also underlined the dependence of the specific experimental paradigm used, the kind of stimuli/information to be integrated, as well as the specific modalities taxed. This is echoed by the finding that older listeners apparently show an intact integration ability of speech stimuli at a phoneme-level, but show compromised integration of whole words [104].

This might also explain supposedly inconsistent findings e.g., in aging-related multisensory integration research [for reviews see $[45,46,96]$ and stresses the importance of interpreting results of audiovisual integration at a task-specific level, in contrast to deriving generalizations from one specific paradigm.

\section{Audiovisual speech-in-noise recognition}

When measuring speech recognition in noise, our expectations were confirmed in that normal-hearing listeners had better SRTs in both auditory-only and audiovisual conditions compared to hearing-impaired listeners. Likewise expectedly, both groups showed an audiovisual benefit over the auditory-only condition. At the same time, both groups displayed the same amount of visual gain as defined by the difference between the $\mathrm{AO}$ and $\mathrm{AV}$ condition. Hence, the groups benefitted equally from additional visual information.

It is well documented that providing additional visual- beyond auditory information, and corresponding integration of these, improves speech intelligibility, especially in adverse listening conditions such as noise [e.g., 2, 5-8, 10, 23, 105, 106]. A benefit from audiovisual compared to auditory-only presentations of sentences has, inter alia, been shown for CI patients [e.g., 107] and mildly hearing-impaired listeners [e.g., 14], but also for normal-hearing listeners [e.g., 2]. Our findings add to previous ones similarly reporting a poorer performance of $\mathrm{HI}$ listeners compared to NH listeners in both auditoryonly and audiovisual conditions $[108,109]$.

When it comes to the relative use of visual information by $\mathrm{HI}$ and $\mathrm{NH}$ individuals, results are mixed: Some studies found hearing-impaired individuals to be more sensitive to visual phonetic information in visual speech perception and to display higher speechreading abilities $[110,111]$ and to make more use of the visual modality compared to $\mathrm{NH}$ 
adults [112-115], whereas others did not find differences in lip-reading abilities $[9,116]$. While one could expect HI individuals to display a higher audiovisual gain compared to NH listeners [cf. 117], our result echoes the findings by Tye-Murray et al. [9], who likewise reported no significant differences in the visual or auditory enhancement between $\mathrm{NH}$ and HI listeners, neither on consonant -, word -, nor sentence level. Correspondingly, Rosemann and Thiel [49] found a similar audiovisual benefit for their NH and HI subjects in an audiovisual sentence task.

As the auditory component likely remains the primary source in audiovisual speech recognition, not only for normal hearing [118] but also in hearing impairment [119-121], audiovisual speech intelligibility of $\mathrm{HI}$ individuals should likewise decrease with degraded auditory input compared to NH listeners - yet, only when constant or fixed SNRs or SPLS are used [cf. 108, 109]. Moradi and colleagues [122] assume that if SNRs or SPLs are individually set, as in Tye-Murray's [9] or Rosemann and Thiel's study [49], the difference in $\mathrm{AV}$ speech recognition between $\mathrm{NH}$ and $\mathrm{HI}$ listeners would cancel out. We similarly used adaptive SNRs, obtaining the individual's signal-to-noise ratio where $80 \%$ of the speech was understood, which might explain the equal audiovisual benefit for the two groups.

On a related note, results of a study by Puschmann et al. [48] point towards the possibility that changes in audiovisual processing in hearing-impaired individuals may only appear for incongruent, conflicting information from the auditory and visual modality rather than for congruent audiovisual input. This might also clarify why we found differences between $\mathrm{NH}$ and $\mathrm{HI}$ individuals in the McGurk test using incongruent audiovisual speech stimuli, but not in the AV-SRT test presenting congruent audiovisual speech information.

Lastly, measurements in terms of SRTs do not reflect the effort a listener uses to obtain that SRT [cf. 123-125]. It is possible that our groups do not exhibit a numerical difference in the audiovisual gain but differ in the amount of listening effort.

Summing up, we found that both HI and NH individuals showed an AV benefit over AO conditions, which is in line with the broad literature on the AV gain, although groups did not differ in their gain from the additional visual information. 
860 Despite the evidence for altered audiovisual processing in elderly adults with hearing 861 impairment, the perceptual effects, e.g., for speech recognition, had previously not been 862 adequately examined. In a last step, we therefore linked audiovisual integration 863 capacities, as determined by the susceptibility to the flash- and McGurk illusion, to 864 audiovisual speech intelligibility in noise, to explore whether and to what extent, variance 865 in AV SiN recognition can be explained by variables of AV integration - beyond age and 866 hearing loss.

The stepwise regression revealed group differences: In the NH group, only one third 868 of the overall variance in AV speech intelligibility was explained by age and audiovisual 869 integration (McGurk). In contrast, more overall variance was explained for the HI group, not only by hearing loss but also by both measures of AVI, which accounted for almost half of the overall variance. Specifically, those with fewer flash illusions and more McGurk illusions had lower AV SRTs and thus better audiovisual speech-in-noise recognition at less favorable SNRs.

A plethora of studies has investigated the differential role of various factors in speechin-noise prediction. Recapitulating, age and hearing thresholds have been reliably identified as good predictors across various studies [20, 126-129, for review see 19]. Simultaneously, however, it became apparent that those measures fail to comprehensively explain the variance in speech recognition, especially in hearingimpaired listeners and under adverse listening conditions such as in noise. Houtgast and Festen [20] emphasized that the hitherto investigated measures of auditory and cognitive abilities still fail to comprehensively account for the variability in SiN recognition, with about $30 \%$ missing. Hence, not all factors crucial for speech-in-noise recognition had been investigated up to that point.

We aimed at filling that gap by investigating the influence of audiovisual integration 885 capacities on specifically audiovisual speech intelligibility in noise, given the multisensory nature of speech and the fact that listeners vary in their usage of visual speech information $887[9,14,16]$. We found that AVI abilities account for additional variance in AV speech-in888 noise recognition, especially for hearing-impaired individuals. So far, no study has 
investigated the influence of two tests of audiovisual integration on audiovisual SiN intelligibility. Grant and Seitz [15] linked the McGurk effect to the audiovisual benefit for consonants and sentences in participants with mild-to severe hearing loss, reporting a positive correlation, whereas in their regression model, the susceptibility to the McGurk effect was not selected as predictor variable for the audiovisual benefit. Van Engen and colleagues [130] added to this by investigating the effect of AV integration, as indexed by the McGurk effect, to audiovisual sentence recognition (comparable to our outcome measure), likewise not finding a relationship and therefore arguing that the McGurk effect may not reflect a valid and relevant measure for audiovisual speech processing. While at first glance their findings seem to contradict ours, they investigated young, normalhearing individuals. It is conceivable, that the influence of AVI abilities on AV speech-innoise intelligibility only emerges when unisensory abilities are compromised as in aging in general and in hearing impairment in particular. This might also explain why the predictive value of both tests of AVI is larger in our HI group than in our NH group.

At the same time, it should be emphasized that in the NH group, only the McGurk tests emerged as significant predictor, whereas in the HI group, the susceptibility to the flash illusion was selected before the McGurk susceptibility. Given that both tests were not correlated, they seem to contribute independently to speech-in-noise recognition, which is also supported by the fact that the coefficients of tests were opposite. Taken together, these results again underline the previous statement that the two tests of audiovisual integration might reflect two distinct aspects of audiovisual processing and can rather complement each other rather than being used interchangeably.

\section{Limitations}

Some limitations of this study should be pointed out, such as the observational character of the study. Although our groups were closely matched in terms of age and were screened for cognitive abilities, we cannot rule out that they differed in another systematic way besides hearing loss, e.g., in specific cognitive domains. Furthermore, we focused on a group with mild hearing impairment, wherefore our results cannot be generalized to more severe forms of hearing loss nor to treated hearing loss (with CIs or hearing aids). Although we aimed at assessing the influence of hearing impairment, a younger control group would have helped to separate age effects that might have added 
to the equivocal findings in multisensory integration research. Furthermore, the high variability of the NH group in the AV gain and in the AV condition, being only partly explained by our included measures, points towards other factors that are particularly important in normal-hearing individuals for audiovisual SiN recognition, such as, for example, cognitive abilities. At the same time, it remains to be elucidated what underlies the variability in AVI abilities. The use of a different frequency in the flash illusion complicates a direct comparison between our previous (Gieseler et al., 2018) and the current study. Furthermore, in the McGurk task, we used a three-alternative forced choice approach, while some argue that one should include at least one open-choice set, e.g., 'other', as the response restriction might lead to a biased response distribution [131].

\section{Conclusion}

Our results provide evidence that audiovisual integration abilities are already altered in mild hearing impairment, while the exact direction depends on the specific test used. This suggests that different measures of AVI reflect different integration processes and should thus not be used interchangeably. At the same time, we found that AVI abilities are relevant for audiovisual speech intelligibility in noise, especially for individuals with a hearing loss. We thus conclude that AVI abilities should be considered for future predictions of speech recognition and hearing-aid outcomes, which - in turn - should be measured audiovisually to account for the multisensory nature of speech and communication, and improve the ecological validity. The finding that AVI abilities are related to SiN intelligibility moreover prompts the idea that training of AVI abilities may improve speech intelligibility under challenging listening conditions.

\section{Acknowledgements}

The authors would like to thank the Hörzentrum Oldenburg GmbH for the collaboration. We particularly thank Meike Renken and Anja Schröck for part of the data collection of the hearing-impaired group as well as Melanie Zokoll, Matthias Vormann, and Niklas Grunewald for their support and valuable comments. We also thank Gerard Llorach, Giso Grimm, Frederike Kirschner, and Volker Hohmann of the Department of Medical Physics (University of Oldenburg) for making available for us their audiovisual version of the OLSA. 
$949 \quad$ Funding

950 This work was supported by the Hearing Industry Research Consortium (IRC) Grant 2017

951 and the Deutsche Forschungsgemeinschaft (DFG, German Research Foundation) under

952 Germany's Excellence Strategy - EXC 2177/1 "Hearing4all" (Project ID 390895286).

\section{$953 \quad$ Conflict of Interest Statement}

954 The authors declare that they have no conflict of interest.

\section{Compliance with Ethical Standard section}

956 Approval for the study was obtained from the Research Ethics Committee of the

957 University of Oldenburg (no 25/2018) and the study was carried out in accordance with 958 the ethical standards of with the Declaration of Helsinki. All participants gave their 959 written informed consent prior to the study.

\section{$960 \quad$ Author Contributions}

961 AG, MT, and HC formulated the overall research question; AG conceptualized the specific 962 research question. AG, MT, SR, CT, KW, and HC contributed to the study design and 963 experimental set-up. AG carried out the experiments together with the Hörzentrum 964 Oldenburg GmbH under the responsibility of KW. AG analyzed the data and wrote the 965 manuscript. MT, SR, CT, KW, and HC critically reviewed and significantly contributed to the 966 manuscript. 


\section{References}

968 1. Picheny MA, Durlach NI, Braida LD. Speaking clearly for the hard of hearing. II:

969 Acoustic characteristics of clear and conversational speech. J Speech Hear Res. 970 1986;29(4):434-46. PubMed PMID: 3795886.

971 2. Sumby WH, Pollack I. Visual Contribution to Speech Intelligibility in Noise. J Acoust

972 Soc Am. 1954;26(2):212-5. doi: Doi 10.1121/1.1907309. PubMed PMID:

973 WOS:A1954UD61600011.

974 3. Erber NP. Visual Perception of Speech by Deaf Children - Recent Developments and 975 Continuing Needs. J Speech Hear Disord. 1974;39(2):178-85. doi: DOI 976 10.1044/jshd.3902.178. PubMed PMID: WOS:A1974T055500008.

977 4. MacLeod A, Summerfield Q. Quantifying the contribution of vision to speech 978 perception in noise. Br J Audiol. 1987;21(2):131-41. PubMed PMID: 3594015.

979 5. Summerfield Q. Lipreading and audio-visual speech perception. Philos Trans R Soc 980 Lond B Biol Sci. 1992;335(1273):71-8. doi: 10.1098/rstb.1992.0009. PubMed PMID: 9811348140.

982 6. Grant KW, Seitz PF. The use of visible speech cues for improving auditory detection 983 of spoken sentences. J Acoust Soc Am. 2000;108(3 Pt 1):1197-208. doi: 984 10.1121/1.1288668. PubMed PMID: 11008820.

985 7. Bernstein LE, Auer ET, Takayanagi S. Auditory speech detection in noise enhanced 986 by lipreading. Speech Commun. 2004;44(1-4):5-18. doi: 10.1016/j.specom.2004.10.011. 987 PubMed PMID: WOS:000226074500002.

988 8. Ross LA, Saint-Amour D, Leavitt VM, Javitt DC, Foxe JJ. Do you see what I am saying? 989 Exploring visual enhancement of speech comprehension in noisy environments. Cereb 990 Cortex. 2007;17(5):1147-53. doi: 10.1093/cercor/bhl024. PubMed PMID: 16785256.

991 9. Tye-Murray N, Sommers MS, Spehar B. Audiovisual integration and lipreading 992 abilities of older adults with normal and impaired hearing. Ear Hear. 2007;28(5):656-68. 993 doi: 10.1097/AUD.0b013e31812f7185. PubMed PMID: 17804980.

994 10. Drijvers L, Ozyurek A. Visual Context Enhanced: The Joint Contribution of Iconic 995 Gestures and Visible Speech to Degraded Speech Comprehension. J Speech Lang Hear R. 996 2017;60(1):212-22. doi: 10.1044/2016_Jslhr-H-16-0101. PubMed PMID: 997 WOS:000392280200017.

998 11. van Wassenhove V, Grant KW, Poeppel D. Visual speech speeds up the neural 999 processing of auditory speech. Proc Natl Acad Sci U S A. 2005;102(4):1181-6. doi: 
1000

1001

1002

1003

1004

1005

1006

1007

1008

1009

1010

1011

1012

1013

1014

1015

1016

1017

1018

1019

1020

1021

1022

1023

1024

1025

1026

1027

1028

1029

1030

1031

10.1073/pnas.0408949102. PubMed PMID: 15647358; PubMed Central PMCID: PMCPMC545853.

12. Arnal LH, Morillon B, Kell CA, Giraud AL. Dual neural routing of visual facilitation in speech processing. J Neurosci. 2009;29(43):13445-53. doi: 10.1523/JNEUROSCI.319409.2009. PubMed PMID: 19864557.

13. Fraser S, Gagne JP, Alepins M, Dubois P. Evaluating the effort expended to understand speech in noise using a dual-task paradigm: the effects of providing visual speech cues. J Speech Lang Hear Res. 2010;53(1):18-33. doi: 10.1044/10924388(2009/08-0140). PubMed PMID: 19635945.

14. Grant KW, Walden BE, Seitz PF. Auditory-visual speech recognition by hearingimpaired subjects: consonant recognition, sentence recognition, and auditory-visual integration. J Acoust Soc Am. 1998;103(5 Pt 1):2677-90. doi: 10.1121/1.422788. PubMed PMID: 9604361.

15. Grant KW, Seitz PF. Measures of auditory-visual integration in nonsense syllables and sentences. J Acoust Soc Am. 1998;104(4):2438-50. doi: 10.1121/1.423751. PubMed PMID: 10491705.

16. Sommers MS, Tye-Murray N, Spehar B. Auditory-visual speech perception and auditory-visual enhancement in normal-hearing younger and older adults. Ear Hear. 2005;26(3):263-75. PubMed PMID: 15937408.

17. Colonius H, Diederich A. A Measure of Auditory-Visual Integration Efficiency Based on Fechnerian Scaling. Auditory-Visual Speech Processing 2007 (AVSP2007); Hilvarenbeek, The Netherlands2007.

18. Altieri N, Hudock D. Assessing variability in audiovisual speech integration skills using capacity and accuracy measures. Int J Audiol. 2014;53(10):710-8. doi: 10.3109/14992027.2014.909053. PubMed PMID: 24806080.

19. Akeroyd MA. Are individual differences in speech reception related to individual differences in cognitive ability? A survey of twenty experimental studies with normal and hearing-impaired adults. Int J Audiol. 2008;47 Suppl 2:S53-71. doi: 10.1080/14992020802301142. PubMed PMID: 19012113.

20. Houtgast T, Festen JM. On the auditory and cognitive functions that may explain an individual's elevation of the speech reception threshold in noise. Int J Audiol. 2008;47(6):287-95. doi: 10.1080/14992020802127109. PubMed PMID: 18569101. 
1032 21. Altieri N, Hudock D. Hearing impairment and audiovisual speech integration 1033 ability: a case study report. Front Psychol. 2014;5:678. doi: 10.3389/fpsyg.2014.00678. 1034 PubMed PMID: 25071649; PubMed Central PMCID: PMCPMC4076931.

1035 22. Peelle JE, Sommers MS. Prediction and constraint in audiovisual speech 1036 perception. Cortex. 2015;68:169-81. doi: 10.1016/j.cortex.2015.03.006. PubMed PMID: 1037 25890390; PubMed Central PMCID: PMCPMC4475441.

1038 23. Rosenblum LD. Speech Perception as a Multimodal Phenomenon. Curr Dir Psychol 1039 Sci. 2008;17(6):405-9. doi: 10.1111/j.1467-8721.2008.00615.x. PubMed PMID: 1040 23914077; PubMed Central PMCID: PMCPMC3732050.

1041 24. Grant KW, Bernstein JGW. Toward a Model of Auditory-Visual Speech 1042 Intelligibility. In: Lee AKC, Wallace MT, Coffin AB, Popper AN, Fay RR, editors. 1043 Multisensory Processes The Auditory Perspective. Springer Handbook of Auditory 1044 Research. 68. Switzerland: Springer Nature Switzerland AG; 2019. p. 33-58.

1045 25. Altieri N. Audiovisual integration: an introduction to behavioral and neuro1046 cognitive methods. Front Psychol. 2013;4:642. doi: 10.3389/fpsyg.2013.00642. PubMed 1047 PMID: 24062715; PubMed Central PMCID: PMCPMC3775457.

1048 26. McGurk H, MacDonald J. Hearing lips and seeing voices. Nature. 1049 1976;264(5588):746-8. doi: 10.1038/264746a0. PubMed PMID: 1012311.

1050 27. Shams L, Kamitani Y, Shimojo S. Illusions. What you see is what you hear. Nature. 1051 2000;408(6814):788. doi: 10.1038/35048669. PubMed PMID: 11130706.

1052 28. Shams L, Kamitani Y, Shimojo S. Visual illusion induced by sound. Brain Res Cogn 1053 Brain Res. 2002;14(1):147-52. PubMed PMID: 12063138.

1054 29. Spence C, Squire S. Multisensory integration: maintaining the perception of 1055 synchrony. Curr Biol. 2003;13(13):R519-21. PubMed PMID: 12842029.

1056 30. van Wassenhove V, Grant KW, Poeppel D. Temporal window of integration in 1057 auditory-visual speech perception. Neuropsychologia. 2007;45(3):598-607. doi: 1058 10.1016/j.neuropsychologia.2006.01.001. PubMed PMID: 16530232.

1059 31. Stevenson RA, Wallace MT. Multisensory temporal integration: task and stimulus 1060 dependencies. Exp Brain Res. 2013;227(2):249-61. doi: 10.1007/s00221-013-3507-3. 1061 PubMed PMID: 23604624; PubMed Central PMCID: PMCPMC3711231.

1062 32. Wallace MT, Stevenson RA. The construct of the multisensory temporal binding 1063 window and its dysregulation in developmental disabilities. Neuropsychologia. 
1064 2014;64:105-23. doi: 10.1016/j.neuropsychologia.2014.08.005. PubMed PMID: 1065 25128432; PubMed Central PMCID: PMCPMC4326640.

1066 33. Vroomen J, Keetels M. Perception of intersensory synchrony: a tutorial review. 1067 Atten Percept Psychophys. 2010;72(4):871-84. doi: 10.3758/APP.72.4.871. PubMed 1068 PMID: 20436185.

1069 34. Stevenson RA, Zemtsov RK, Wallace MT. Individual differences in the multisensory 1070 temporal binding window predict susceptibility to audiovisual illusions. J Exp Psychol 1071 Hum Percept Perform. 2012;38(6):1517-29. doi: 10.1037/a0027339. PubMed PMID: 1072 22390292; PubMed Central PMCID: PMCPMC3795069.

1073 35. Calvert GA. Crossmodal processing in the human brain: insights from functional 1074 neuroimaging studies. Cereb Cortex. 2001;11(12):1110-23. doi: 1075 10.1093/cercor/11.12.1110. PubMed PMID: 11709482.

1076 36. Laurienti PJ, Burdette JH, Maldjian JA, Wallace MT. Enhanced multisensory 1077 integration in older adults. Neurobiol Aging. 2006;27(8):1155-63. doi: 1078 10.1016/j.neurobiolaging.2005.05.024. PubMed PMID: 16039016.

1079 37. Peiffer AM, Mozolic JL, Hugenschmidt CE, Laurienti PJ. Age-related multisensory 1080 enhancement in a simple audiovisual detection task. Neuroreport. 2007;18(10):1077-81. 1081 doi: 10.1097/WNR.0b013e3281e72ae7. PubMed PMID: 17558300.

1082 38. Diederich A, Colonius H, Schomburg A. Assessing age-related multisensory 1083 enhancement with the time-window-of-integration model. Neuropsychologia. 1084 2008;46(10):2556-62. doi: 10.1016/j.neuropsychologia.2008.03.026. PubMed PMID: 108518490033.

1086 39. Mahoney JR, Li PC, Oh-Park M, Verghese J, Holtzer R. Multisensory integration 1087 across the senses in young and old adults. Brain Res. 2011;1426:43-53. doi: 1088 10.1016/j.brainres.2011.09.017. PubMed PMID: 22024545; PubMed Central PMCID: 1089 PMCРMC3232048.

1090 40. DeLoss DJ, Pierce RS, Andersen GJ. Multisensory integration, aging, and the sound1091 induced flash illusion. Psychol Aging. 2013;28(3):802-12. doi: 10.1037/a0033289. 1092 PubMed PMID: 23978009; PubMed Central PMCID: PMCPMC3778128.

1093 41. Setti A, Burke KE, Kenny R, Newell FN. Susceptibility to a multisensory speech 1094 illusion in older persons is driven by perceptual processes. Front Psychol. 2013;4:575. 1095 doi: 10.3389/fpsyg.2013.00575. PubMed PMID: 24027544; PubMed Central PMCID: 1096 PMCРMC3760087. 
1097 42. Tye-Murray N, Sommers M, Spehar B, Myerson J, Hale S. Aging, audiovisual 1098 integration, and the principle of inverse effectiveness. Ear Hear. 2010;31(5):636-44. doi: 1099 10.1097/AUD.0b013e3181ddf7ff. PubMed PMID: 20473178; PubMed Central PMCID: 1100 PMCPMC2924437.

1101 43. Roudaia E, Sekuler AB, Bennett PJ, Sekuler R. Aging and audio-visual and multi-cue 1102 integration in motion. Front Psychol. 2013;4:267. doi: 10.3389/fpsyg.2013.00267. 1103 PubMed PMID: 23734132; PubMed Central PMCID: PMCPMC3661954.

1104 44. Huyse A, Leybaert J, Berthommier F. Effects of aging on audio-visual speech 1105 integration. J Acoust Soc Am. 2014;136(4):1918-31. doi: 10.1121/1.4894685. PubMed 1106 PMID: 25324091.

1107 45. Mozolic JL, Hugenschmidt CE, Peiffer AM, Laurienti PJ. Multisensory Integration 1108 and Aging. In: Murray MM, Wallace MT, editors. The Neural Bases of Multisensory 1109 Processes. Frontiers in Neuroscience. Boca Raton (FL)2012.

1110 46. de Dieuleveult AL, Siemonsma PC, van Erp JB, Brouwer AM. Effects of Aging in 1111 Multisensory Integration: A Systematic Review. Front Aging Neurosci. 2017;9:80. doi: 1112 10.3389/fnagi.2017.00080. PubMed PMID: 28400727; PubMed Central PMCID: 1113 PMCРMC5368230.

1114 47. Musacchia G, Arum L, Nicol T, Garstecki D, Kraus N. Audiovisual deficits in older 1115 adults with hearing loss: biological evidence. Ear Hear. 2009;30(5):505-14. doi: 1116 10.1097/AUD.0b013e3181a7f5b7. PubMed PMID: 19546807.

1117 48. Puschmann S, Sandmann P, Bendixen A, Thiel CM. Age-related hearing loss 1118 increases cross-modal distractibility. Hear Res. 2014;316:28-36. doi: 1119 10.1016/j.heares.2014.07.005. PubMed PMID: 25080386.

1120 49. Rosemann S, Thiel CM. Audio-visual speech processing in age-related hearing loss: 1121 Stronger integration and increased frontal lobe recruitment. Neuroimage. 2018;175:4251122 37. doi: 10.1016/j.neuroimage.2018.04.023. PubMed PMID: 29655940.

1123 50. Barone P, Deguine O. Multisensory Processing in Cochlear Implant Listeners. In: 1124 Zeng F-G, Popper AN, Fay RR, editors. Auditory Prostheses: New Horizons. Springer 1125 Handbook of Auditory Research. 39. New York: Springer'; 2012. p. 365-81.

1126 51. Stevenson RA, Sheffield SW, Butera IM, Gifford RH, Wallace MT. Multisensory 1127 Integration in Cochlear Implant Recipients. Ear Hear. 2017;38(5):521-38. doi: 1128 10.1097/AUD.0000000000000435. PubMed PMID: 28399064; PubMed Central PMCID: 1129 PMCPMC5570631. 
1130 52. Gieseler A, Tahden MAS, Thiel CM, Colonius H. Does hearing aid use affect 1131 audiovisual integration in mild hearing impairment? Exp Brain Res. 2018;236(4):11611132 79. doi: 10.1007/s00221-018-5206-6. PubMed PMID: 29453491.

1133 53. Llorach G, Kirschner F, Grimm G, Zokoll MA, Wagener KC, Hohmann V. 1134 Development and Evaluation of Video Recordings for the OLSA Matrix Sentence Test. 1135 2019. doi: arXiv:1912.04700v1.

1136 54. (PBD) WPoBaD. Grades of hearing impairment 1137 https://www.who.int/pbd/deafness/hearing_impairment_grades/ [cited 2016 March 1138 14th].

1139 55. von Gablenz P, Holube I. [Prevalence of hearing impairment in northwestern 1140 Germany. Results of an epidemiological study on hearing status (HORSTAT)]. HNO. 1141 2015;63(3):195-214. doi: 10.1007/s00106-014-2949-7. PubMed PMID: 25720301.

1142 56. Kalbe E, Kessler J, Calabrese P, Smith R, Passmore AP, Brand M, et al. DemTect: a 1143 new, sensitive cognitive screening test to support the diagnosis of mild cognitive 1144 impairment and early dementia. Int J Geriatr Psychiatry. 2004;19(2):136-43. doi: 1145 10.1002/gps.1042. PubMed PMID: 14758579.

1146 57. Stropahl M, Schellhardt S, Debener S. McGurk stimuli for the investigation of 1147 multisensory integration in cochlear implant users: The Oldenburg Audio Visual Speech 1148 Stimuli (OLAVS). Psychon Bull Rev. 2017;24(3):863-72. doi: 10.3758/s13423-016-11481149 9. PubMed PMID: 27562763.

1150 58. Grimm G, Llorach G, Hendrikse MME, Hohmann V, editors. Audio-visual stimuli for 1151 the evaluation of speech-enhancing algorithms. Proceedings of the 23rd International 1152 Congress on Acoustics; 2019; Aachen, Germany.

1153 59. Llorach G, Hohmann V, editors. Word error and confusion patterns in an 1154 audiovisual German matrix sentence test (OLSA). Proceedings of the 23rd International 1155 Congress on Acoustics; 2019; Aachen, Germany.

1156 60. Wagener KC, Brand T, Kollmeier B. Entwicklung und Evaluation eines Satztests für 1157 die deutsche Sprache II: Optimierung des Oldenburger Satztests [Development and 1158 evaluation of a German sentence test II: Optimization of the Oldenburg sentence test]. 1159 Zeitschrift für Audiologie. 1999;38:44-56.

1160 61. Wagener KC, Brand T, Kollmeier B. Entwicklung und Evaluation eines Satztests in 1161 deutscher Sprache III: Evaluation des Oldenburger Satztests [Development and 
1162 evaluation of a German sentence test III: Evaluation of the Oldenburg sentence test]. 1163 Zeitschrift für Audiologie. 1999;38:86-95.

1164 62. Wagener KC, Kühnel V, Kollmeier B. Entwicklung und Evaluation eines Satztests 1165 für die deutsche Sprache I: Design des Oldenburger Satztests [Development and 1166 evaluation of a German sentence test I: Design of the Oldenburg sentence test]. Zeitschrift 1167 für Audiologie. 1999;38:4-15.

1168 63. Brand T, Wagener KC. Eigenschaften, Leistungen und Grenzen von Matrixtests. 1169 HNO. 2017;65(3):182-8.

1170 64. Dreschler WA, Verschuure H, Ludvigsen C, Westermann S. ICRA noises: artificial 1171 noise signals with speech-like spectral and temporal properties for hearing instrument 1172 assessment. International Collegium for Rehabilitative Audiology. Audiology. 1173 2001;40(3):148-57. PubMed PMID: 11465297.

1174 65. Brand T, Kollmeier B. Efficient adaptive procedures for threshold and concurrent 1175 slope estimates for psychophysics and speech intelligibility tests. J Acoust Soc Am. 1176 2002;111(6):2801-10. doi: 10.1121/1.1479152. PubMed PMID: 12083215.

1177 66. Foss-Feig JH, Kwakye LD, Cascio CJ, Burnette CP, Kadivar H, Stone WL, et al. An 1178 extended multisensory temporal binding window in autism spectrum disorders. Exp 1179 Brain Res. 2010;203(2):381-9. doi: 10.1007/s00221-010-2240-4. PubMed PMID: 1180 20390256; PubMed Central PMCID: PMCPMC2871100.

1181 67. MacDonald J, McGurk H. Visual influences on speech perception processes. Percept 1182 Psychophys. 1978;24(3):253-7. PubMed PMID: 704285.

1183 68. Hugenschmidt CE, Peiffer AM, McCoy TP, Hayasaka S, Laurienti PJ. Preservation of 1184 crossmodal selective attention in healthy aging. Exp Brain Res. 2009;198(2-3):273-85. 1185 doi: 10.1007/s00221-009-1816-3. PubMed PMID: 19404621; PubMed Central PMCID: 1186 PMCPMC2952884.

1187 69. McGovern DP, Roudaia E, Stapleton J, McGinnity TM, Newell FN. The sound1188 induced flash illusion reveals dissociable age-related effects in multisensory integration. 1189 Front Aging Neurosci. 2014;6:250. doi: 10.3389/fnagi.2014.00250. PubMed PMID: 1190 25309430; PubMed Central PMCID: PMCPMC4174115.

1191 70. Stevenson RA, Baum SH, Krueger J, Newhouse PA, Wallace MT. Links between 1192 temporal acuity and multisensory integration across life span. J Exp Psychol Hum Percept 1193 Perform. 2018;44(1):106-16. doi: 10.1037/xhp0000424. PubMed PMID: 28447850; 1194 PubMed Central PMCID: PMCPMC5659980. 
1195 71. Stropahl M, Debener S. Auditory cross-modal reorganization in cochlear implant 1196 users indicates audio-visual integration. Neuroimage Clin. 2017;16:514-23. doi: 1197 10.1016/j.nicl.2017.09.001. PubMed PMID: 28971005; PubMed Central PMCID: 1198 PMCPMC5609862.

1199 72. Massaro DW, Cohen MM. The paradigm and the fuzzy logical model of perception 1200 are alive and well. J Exp Psychol Gen. 1993;122(1):115-24. PubMed PMID: 8440975.

1201 73. Munhall KG, Gribble P, Sacco L, Ward M. Temporal constraints on the McGurk 1202 effect. Percept Psychophys. 1996;58(3):351-62. PubMed PMID: 8935896.

1203 74. Hillock AR, Powers AR, Wallace MT. Binding of sights and sounds: age-related 1204 changes in multisensory temporal processing. Neuropsychologia. 2011;49(3):461-7. doi: 1205 10.1016/j.neuropsychologia.2010.11.041. PubMed PMID: 21134385; PubMed Central 1206 PMCID: PMCPMC3140703.

1207 75. Miller LM, D'Esposito M. Perceptual fusion and stimulus coincidence in the cross1208 modal integration of speech. J Neurosci. 2005;25(25):5884-93. doi: 1209 10.1523/JNEUROSCI.0896-05.2005. PubMed PMID: 15976077.

1210 76. Powers AR, 3rd, Hillock AR, Wallace MT. Perceptual training narrows the temporal 1211 window of multisensory binding. J Neurosci. 2009;29(39):12265-74. doi: 1212 10.1523/JNEUROSCI.3501-09.2009. PubMed PMID: 19793985; PubMed Central PMCID: 1213 PMCPMC2771316.

1214 77. Zampini M, Shore DI, Spence C. Audiovisual temporal order judgments. Exp Brain 1215 Res. 2003;152(2):198-210. doi: 10.1007/s00221-003-1536-z. PubMed PMID: 12879178.

1216 78. Desai S, Stickney G, Zeng FG. Auditory-visual speech perception in normal-hearing 1217 and cochlear-implant listeners. J Acoust Soc Am. 2008;123(1):428-40. doi: 1218 10.1121/1.2816573. PubMed PMID: 18177171; PubMed Central PMCID: 1219 PMCPMC2662523.

1220 79. Huyse A, Berthommier F, Leybaert J. Degradation of labial information modifies 1221 audiovisual speech perception in cochlear-implanted children. Ear Hear. 2013;34(1):1101222 21. doi: 10.1097/AUD.0b013e3182670993. PubMed PMID: 23059850.

1223 80. Rouger J, Fraysse B, Deguine O, Barone P. McGurk effects in cochlear-implanted 1224 deaf subjects. Brain Res. 2008;1188:87-99. doi: 10.1016/j.brainres.2007.10.049. PubMed 1225 PMID: 18062941.

1226 81. Schorr EA, Fox NA, van Wassenhove V, Knudsen EI. Auditory-visual fusion in 1227 speech perception in children with cochlear implants. Proc Natl Acad Sci U S A. 
2005;102(51):18748-50. doi: 10.1073/pnas.0508862102. PubMed PMID: 16339316; PubMed Central PMCID: PMCPMC1317952.

1230 82. Tremblay C, Champoux F, Lepore F, Theoret H. Audiovisual fusion and cochlear 1231 implant proficiency. Restor Neurol Neurosci. 2010;28(2):283-91. doi: 10.3233/RNN1232 2010-0498. PubMed PMID: 20404415.

1233 83. Alais D, Burr D. The ventriloquist effect results from near-optimal bimodal 1234 integration. Curr Biol. 2004;14(3):257-62. doi: 10.1016/j.cub.2004.01.029. PubMed 1235 PMID: 14761661.

1236 84. Ernst MO, Banks MS. Humans integrate visual and haptic information in a 1237 statistically optimal fashion. Nature. 2002;415(6870):429-33. doi: 10.1038/415429a. 1238 PubMed PMID: 11807554.

1239 85. Helbig HB, Ernst MO. Optimal integration of shape information from vision and 1240 touch. Exp Brain Res. 2007;179(4):595-606. doi: 10.1007/s00221-006-0814-y. PubMed 1241 PMID: 17225091.

1242 86. Colonius H, Diederich A. Formal models and quantitative measures of multisensory 1243 integration: a selective overview. Eur J Neurosci. 2017. doi: 10.1111/ejn.13813. PubMed 1244 PMID: 29285815.

1245 87. Setti A, Burke KE, Kenny RA, Newell FN. Is inefficient multisensory processing 1246 associated with falls in older people? Exp Brain Res. 2011;209(3):375-84. doi: 1247 10.1007/s00221-011-2560-z. PubMed PMID: 21293851.

1248 88. Chan JS, Kaiser J, Brandl M, Matura S, Prvulovic D, Hogan MJ, et al. Expanded 1249 temporal binding windows in people with mild cognitive impairment. Curr Alzheimer Res. 1250 2015;12(1):61-8. PubMed PMID: 25523426.

1251 89. Keil J, Muller N, Hartmann T, Weisz N. Prestimulus beta power and phase 1252 synchrony influence the sound-induced flash illusion. Cereb Cortex. 2014;24(5):1278-88. 1253 doi: 10.1093/cercor/bhs409. PubMed PMID: 23300109.

1254 90. Mishra J, Martinez A, Hillyard SA. Cortical processes underlying sound-induced 1255 flash fusion. Brain Res. 2008;1242:102-15. doi: 10.1016/j.brainres.2008.05.023. PubMed 1256 PMID: 18585695; PubMed Central PMCID: PMCPMC2584169.

1257 91. Chan YM, Pianta MJ, McKendrick AM. Reduced audiovisual recalibration in the 1258 elderly. Front Aging Neurosci. 2014;6:226. doi: 10.3389/fnagi.2014.00226. PubMed 1259 PMID: 25221508; PubMed Central PMCID: PMCPMC4145582. 
1260 92. Chan YM, Pianta MJ, McKendrick AM. Older age results in difficulties separating 1261 auditory and visual signals in time. J Vis. 2014;14(11). doi: 10.1167/14.11.13. PubMed 1262 PMID: 25253873.

1263 93. Fitzgibbons PJ, Gordon-Salant S. Aging and temporal discrimination in auditory 1264 sequences. J Acoust Soc Am. 2001;109(6):2955-63. doi: 10.1121/1.1371760. PubMed 1265 PMID: 11425137.

1266 94. Gordon-Salant S, Fitzgibbons PJ, Yeni-Komshian GH. Auditory Temporal 1267 Processing and Aging: Implications for Speech Understanding of Older People. Audiol Res. 1268 2011;1(1):e4. doi: 10.4081/audiores.2011.e4. PubMed PMID: 26557313; PubMed Central 1269 PMCID: PMCPMC4627162.

1270 95. Bedard G, Barnett-Cowan M. Impaired timing of audiovisual events in the elderly. 1271 Exp Brain Res. 2016;234(1):331-40. doi: 10.1007/s00221-015-4466-7. PubMed PMID: 127226474576.

1273 96. Baum SH, Stevenson R. Shifts in Audiovisual Processing in Healthy Aging. Curr 1274 Behav Neurosci Rep. 2017;4(3):198-208. doi: 10.1007/s40473-017-0124-7. PubMed 1275 PMID: 29862161; PubMed Central PMCID: PMCPMC5972362.

1276 97. Stevenson RA, Wilson MM, Powers AR, Wallace MT. The effects of visual training 1277 on multisensory temporal processing. Exp Brain Res. 2013;225(4):479-89. doi: 1278 10.1007/s00221-012-3387-y. PubMed PMID: 23307155; PubMed Central PMCID: 1279 PMCPMC3606590.

1280 98. Tremblay C, Champoux F, Voss P, Bacon BA, Lepore F, Theoret H. Speech and non1281 speech audio-visual illusions: a developmental study. PLoS One. 2007;2(1):e742. doi: 1282 10.1371/journal.pone.0000742. PubMed PMID: 17710142; PubMed Central PMCID: 1283 PMCPMC1937019.

1284 99. Tuomainen J, Andersen TS, Tiippana K, Sams M. Audio-visual speech perception is 1285 special. Cognition. 2005;96(1):B13-22. doi: 10.1016/j.cognition.2004.10.004. PubMed 1286 PMID: 15833302.

1287 100. Kaiser J, Hertrich I, Ackermann H, Mathiak K, Lutzenberger W. Hearing lips: 1288 gamma-band activity during audiovisual speech perception. Cereb Cortex. 1289 2005;15(5):646-53. doi: 10.1093/cercor/bhh166. PubMed PMID: 15342432.

1290 101. Shams L, Kamitani Y, Thompson S, Shimojo S. Sound alters visual evoked potentials 1291 in humans. Neuroreport. 2001;12(17):3849-52. PubMed PMID: 11726807. 
1292 102. Shams L, Iwaki S, Chawla A, Bhattacharya J. Early modulation of visual cortex by 1293 sound: an MEG study. Neurosci Lett. 2005;378(2):76-81. doi: 1294 10.1016/j.neulet.2004.12.035. PubMed PMID: 15774261.

1295 103. Soto-Faraco S, Alsius A. Deconstructing the McGurk-MacDonald illusion. J Exp 1296 Psychol Hum Percept Perform. 2009;35(2):580-7. doi: 10.1037/a0013483. PubMed 1297 PMID: 19331510.

1298 104. Stevenson RA, Nelms CE, Baum SH, Zurkovsky L, Barense MD, Newhouse PA, et al. 1299 Deficits in audiovisual speech perception in normal aging emerge at the level of whole1300 word recognition. Neurobiol Aging. 2015;36(1):283-91. doi: 1301 10.1016/j.neurobiolaging.2014.08.003. PubMed PMID: 25282337; PubMed Central 1302 PMCID: PMCPMC4268368.

1303 105. Irwin J, DiBlasi L. Audiovisual speech perception: A new approach and implications 1304 for clinical populations. Lang Linguist Compass. 2017;11(3):77-91. doi: 1305 10.1111/lnc3.12237. PubMed PMID: 29520300; PubMed Central PMCID: 1306 PMCPMC5839512.

1307 106. Campbell R. The processing of audio-visual speech: empirical and neural bases. 1308 Philos Trans R Soc Lond B Biol Sci. 2008;363(1493):1001-10. doi: 1309 10.1098/rstb.2007.2155. PubMed PMID: 17827105; PubMed Central PMCID: 1310 PMCPMC2606792.

1311 107. Hay-McCutcheon MJ, Pisoni DB, Kirk KI. Audiovisual speech perception in elderly 1312 cochlear implant recipients. Laryngoscope. 2005;115(10):1887-94. doi: 1313 10.1097/01.mlg.0000173197.94769.ba. PubMed PMID: 16222216; PubMed Central 1314 PMCID: PMCPMC3315695.

1315 108. Bernstein JG, Grant KW. Auditory and auditory-visual intelligibility of speech in 1316 fluctuating maskers for normal-hearing and hearing-impaired listeners. J Acoust Soc Am. 1317 2009;125(5):3358-72. doi: 10.1121/1.3110132. PubMed PMID: 19425676.

1318 109. Başkent D, Bazo D. Audiovisual asynchrony detection and speech intelligibility in 1319 noise with moderate to severe sensorineural hearing impairment. Ear Hear. 1320 2011;32(5):582-92. doi: 10.1097/AUD.0b013e31820fca23. PubMed PMID: 21389856.

1321 110. Auer ET, Jr., Bernstein LE. Enhanced visual speech perception in individuals with 1322 early-onset hearing impairment. J Speech Lang Hear Res. 2007;50(5):1157-65. doi: 1323 10.1044/1092-4388(2007/080). PubMed PMID: 17905902. 
1324 111. Bernstein LE, Demorest ME, Tucker PE. Speech perception without hearing. 1325 Percept Psychophys. 2000;62(2):233-52. PubMed PMID: 10723205.

1326 112. Rouger J, Lagleyre S, Fraysse B, Deneve S, Deguine O, Barone P. Evidence that 1327 cochlear-implanted deaf patients are better multisensory integrators. Proc Natl Acad Sci 1328 U S A. 2007;104(17):7295-300. doi: 10.1073/pnas.0609419104. PubMed PMID: $132917404220 ;$ PubMed Central PMCID: PMCPMC1855404.

1330 113. Mitchell TV, Maslin MT. How vision matters for individuals with hearing loss. Int J 1331 Audiol. 2007;46(9):500-11. doi: 10.1080/14992020701383050. PubMed PMID: 133217828666.

1333 114. Kaiser AR, Kirk KI, Lachs L, Pisoni DB. Talker and lexical effects on audiovisual 1334 word recognition by adults with cochlear implants. J Speech Lang Hear Res. 1335 2003;46(2):390-404. doi: 10.1044/1092-4388(2003/032). PubMed PMID: 14700380; 1336 PubMed Central PMCID: PMCPMC3432920.

1337 115. Bavelier D, Dye MW, Hauser PC. Do deaf individuals see better? Trends Cogn Sci. 1338 2006;10(11):512-8. doi: 10.1016/j.tics.2006.09.006. PubMed PMID: 17015029; PubMed 1339 Central PMCID: PMCPMC2885708.

1340 116. Lyxell B, Ronnberg J. Information-processing skill and speech-reading. Br J Audiol. 1341 1989;23(4):339-47. PubMed PMID: 2605387.

1342 117. Puschmann S, Daeglau M, Stropahl M, Mirkovic B, Rosemann S, Thiel CM, et al. 1343 Hearing-impaired listeners show increased audiovisual benefit when listening to speech 1344 in noise. Neuroimage. 2019;196:261-8. doi: 10.1016/j.neuroimage.2019.04.017. PubMed 1345 PMID: 30978494.

1346 118. Baart M, Vroomen J, Shaw K, Bortfeld H. Degrading phonetic information affects 1347 matching of audiovisual speech in adults, but not in infants. Cognition. 2014;130(1):311348 43. doi: 10.1016/j.cognition.2013.09.006. PubMed PMID: 24141035; PubMed Central 1349 PMCID: PMCPMC3904288.

1350 119. Picou EM, Ricketts TA, Hornsby BW. How hearing aids, background noise, and 1351 visual cues influence objective listening effort. Ear Hear. 2013;34(5):e52-64. doi: 1352 10.1097/AUD.0b013e31827f0431. PubMed PMID: 23416751.

1353 120. Corthals P, Vinck B, De Vel E, Van Cauwenberge P. Audiovisual speech reception in 1354 noise and self-perceived hearing disability in sensorineural hearing loss. Audiology. 1355 1997;36(1):46-56. PubMed PMID: 9063560. 
1356 121. Corthals P, Vinck B, De Vel E, Van Cauwenberge P. Bilateral threshold frequency 1357 weighting in hearing disability predictions. Scand Audiol. 1997;26(4):211-7. PubMed 1358 PMID: 9428029.

1359 122. Moradi S, Lidestam B, Ronnberg J. Comparison of Gated Audiovisual Speech 1360 Identification in Elderly Hearing Aid Users and Elderly Normal-Hearing Individuals: 1361 Effects of Adding Visual Cues to Auditory Speech Stimuli. Trends in hearing. 2016;20. doi: 10.1177/2331216516653355. PubMed PMID: 27317667. 123. Krueger M, Schulte M, Zokoll MA, Wagener KC, Meis M, Brand T, et al. Relation 1364 Between Listening Effort and Speech Intelligibility in Noise. Am J Audiol. 1365 2017;26(3S):378-92. doi: 10.1044/2017_AJA-16-0136. PubMed PMID: 29049622. 124. Lau MK, Hicks C, Kroll T, Zupancic S. Effect of Auditory Task Type on Physiological and Subjective Measures of Listening Effort in Individuals With Normal Hearing. J Speech Lang Hear Res. 2019;62(5):1549-60. doi: 10.1044/2018_JSLHR-H-17-0473. PubMed PMID: 31063438.

125. Ohlenforst B, Zekveld AA, Jansma EP, Wang Y, Naylor G, Lorens A, et al. Effects of Hearing Impairment and Hearing Aid Amplification on Listening Effort: A Systematic Review. Ear Hear. 2017;38(3):267-81. doi: 10.1097/AUD.0000000000000396. PubMed PMID: 28234670; PubMed Central PMCID: PMCPMC5405775.

1374 126. Humes LE. Factors underlying the speech-recognition performance of elderly 1375 hearing-aid wearers. J Acoust Soc Am. 2002;112(3 Pt 1):1112-32. doi: 10.1121/1.1499132. PubMed PMID: 12243159.

1377 127. Humes LE, Watson BU, Christensen LA, Cokely CG, Halling DC, Lee L. Factors associated with individual differences in clinical measures of speech recognition among the elderly. J Speech Hear Res. 1994;37(2):465-74. PubMed PMID: 8028328. 128. van Rooij JC, Plomp R. Auditive and cognitive factors in speech perception by elderly listeners. II: Multivariate analyses. J Acoust Soc Am. 1990;88(6):2611-24. doi: 10.1121/1.399981. PubMed PMID: 2283434. 129. van Rooij JC, Plomp R. Auditive and cognitive factors in speech perception by 1384 elderly listeners. Acta Otolaryngol Suppl. 1990;476:177-81. PubMed PMID: 2087960.

1385 130. Van Engen KJ, Xie Z, Chandrasekaran B. Audiovisual sentence recognition not 1386 predicted by susceptibility to the McGurk effect. Attention, perception \& psychophysics. 2017;79(2):396-403. doi: 10.3758/s13414-016-1238-9. 
1388 131. Alsius A, Paré M, Munhall KG. Forty Years After Hearing Lips and Seeing Voices: 1389 the McGurk Effect Revisited. 2018;31(1-2):111. doi: 10.1163/22134808-00002565. 